TRANSACTIONS OF THE

AMERICAN MATHEMATICAL SOCIETY

Volume 359, Number 8, August 2007, Pages 3733-3768

S 0002-9947(07)04087-1

Article electronically published on March 7, 2007

\title{
MODULI OF ROOTS OF LINE BUNDLES ON CURVES
}

\author{
LUCIA CAPORASO, CINZIA CASAGRANDE, AND MAURIZIO CORNALBA
}

\begin{abstract}
We treat the problem of completing the moduli space for roots of line bundles on curves. Special attention is devoted to higher spin curves within the universal Picard scheme. Two new different constructions, both using line bundles on nodal curves as boundary points, are carried out and compared with pre-existing ones.
\end{abstract}

\section{INTRODUCTION}

The problem of compactifying the moduli space for roots of line bundles on curves is the object of this paper.

More precisely, consider a family of nodal curves $f: \mathcal{C} \rightarrow B$, with nonsingular fibers over an open subset $U$ of $B$; let $\mathcal{N}$ be a line bundle on $\mathcal{C}$, viewed as a family of line bundles on the fibers of $f$. Assume that the relative degree of $\mathcal{N}$ is divisible by some positive integer $r$. Then the family of $r$-th roots 11 of the restriction of $\mathcal{N}$ to the nonsingular fibers is an étale covering of $U$.

The issue is how to compactify over $B$ such a covering, in a modular way. We define and explore two different approaches, and compare our resulting moduli spaces between themselves and with pre-existing ones.

One of the first cases in which such a question was considered is the one of 2torsion points in the Jacobian, that is, the case $N=\mathcal{O}, r=2$. This was solved by A. Beauville [4] by means of admissible coverings.

Another remarkable, well-known instance is the one of the so-called "higher spin curves", which corresponds to $\mathcal{N}=\omega_{f}$ and, more generally, to $\mathcal{N}=\omega_{f}^{\otimes l}$.

The case of genuine "spin curves", that is, $r=2$ (and $l=1$ ), was solved by Cornalba in [8], where a geometrically meaningful compactification was constructed over the moduli space $\bar{M}_{g}$ of Deligne-Mumford stable curves. The boundary points are certain line bundles on nodal curves.

Later T. J. Jarvis (in 14 and [15) used rank 1 torsion-free sheaves to approach the problem (over stable curves) for all $r$ and $l$. In particular, he constructed two stack compactifications $\overline{\mathfrak{S}}$ and RooT, the first of which is the normalization of the second, and turns out to be smooth. For $r=2$ his compactifications are all isomorphic to the one of 8 .

Other methods have recently been used by D. Abramovich and T. J. Jarvis [1] and by A. Chiodo [7. Both employ (differently) "twisted curves", which, roughly speaking, are nodal curves with a stack structure at some nodes. The resulting compactifications are isomorphic to Jarvis's $\overline{\mathfrak{S}}$.

Received by the editors April 12, 2005 and, in revised form, May 25, 2005.

2000 Mathematics Subject Classification. Primary 14H10, 14H60; Secondary 14K30.

${ }^{1}$ If $L$ and $N$ are line bundles on $X$ such that $L^{\otimes r}=N$, we call $L$ an $r$-th root of $N$. 
Over the last decade, interest in higher spin curves has been revived by the generalized Witten conjecture, which predicts that the intersection theory on their moduli spaces is governed by the Gelfand-Dikil (also known as higher KdV) hierarchy. This conjecture is open 2 see [18] for more details and some recent progress.

The first part of our paper presents a solution of the general problem in the same spirit as [8]; we complete the $r$-th root functor (and its moduli scheme) for any family and any line bundle $\mathcal{N}$ as above, by means of line bundles on nodal curves. For clarity (and to distinguish it from other approaches) we name the resulting space the moduli space for limit roots. Some of the results in this part can already be found, in one form or another, in the existing literature; however, it seemed worthwhile to present a coherent account of the subject based on the ideas of [8]. In order to limit the length of the paper, we work in the context of schemes and coarse moduli spaces, although it is quite clear that our methods lend themselves to the construction of stacks which are fine moduli spaces for the problem at hand. We plan to return to the question in a future paper.

As in the case $r=2$ (see [6]), the combinatorial aspects of our construction play a significant role, and enable us to offer a fairly explicit description of our compactification.

We prove in Theorem 4.2.3 that, in the special case of higher spin curves, our moduli space is isomorphic to the coarse space underlying Jarvis's Root.

The last section is devoted to our second approach, whose goal is to obtain a completion within the compactified Picard scheme, where, obviously, $r$-th roots on smooth curves naturally live. Because of the relative newness of this method, we only apply it to higher spin curves over $\bar{M}_{g}$. A modular compactification of the universal Picard scheme was constructed in [5] by Caporaso, by means of line bundles on semistable curves. This space was later given a description in terms of rank 1 torsion-free sheaves by R. Pandharipande [20], as a special case of a more general result, valid for all ranks.

The question on whether the existing compactifications of higher spin curves embed in the universal Picard scheme seemed, at least to us, irresistible. All the more so because of the strong similarities between the boundary points used by the various authors: (analogous) line bundles in 8 ] and [5] on the one hand, and rank 1 torsion-free sheaves in [14] and 20] on the other.

Therefore we construct and study a new modular compactification of higher spin curves, $\widehat{S}_{g}^{r, l}$, sitting inside the universal Picard scheme, and whose main new feature is that it is not finite over $\bar{M}_{g}$, as soon as $r \geq 3$.

We then prove that, for $r \geq 3$, the previously constructed moduli spaces of limit roots, which we denote by $\bar{S}_{g}^{r, l}$, as well as those constructed in [14, [15], 11 and [7, do not embed in the universal Picard scheme. More precisely, neither is the natural birational map $\chi: \bar{S}_{g}^{r, l} \rightarrow \widehat{S}_{g}^{r, l}$ regular, nor is its inverse (Theorem 5.4 .2 and its corollary). The case $r=2$, treated by C. Fontanari in [12, is an exception, as $\chi$ is regular and bijective.

For higher rank, in the same spirit as ours, a compactification of the moduli space of vector bundles over curves using, instead of torsion-free sheaves, vector bundles over semistable curves, has recently been constructed by A. Schmitt [22],

\footnotetext{
${ }^{2}$ Added in proof. The generalized Witten conjecture has recently been proved by C. Faber, S. Shadrin and D. Zvonkine in Faber, Shadrin, Zvonkine: Tautological relations and the r-spin Witten conjecture. Preprint math.AG/0612510.
} 
continuing upon the work of C. S. Seshadri and others (see [23] and references therein).

1.1. Notation. Throughout the paper we work over the field of complex numbers. We shall use the words "line bundle" as a synonym of "invertible sheaf".

For any scheme $Z$, we denote by $\nu=\nu_{Z}: Z^{\nu} \rightarrow Z$ the normalization morphism.

$C$ will always be a connected, nodal, projective curve of (arithmetic) genus $g$. We denote by $g^{\nu}$ the genus of its normalization.

To $C$ we associate a dual graph $\Gamma_{C}$, as follows. The set of vertices of $\Gamma_{C}$ is the set of components of $C^{\nu}$. The set of half-edges is the set of all points of $C^{\nu}$ mapping to a node of $C$. Two half-edges form an edge of $\Gamma_{C}$ when the two corresponding points map to the same node of $C$. Recall that $g=g^{\nu}+b_{1}\left(\Gamma_{C}\right)$, where $b_{1}\left(\Gamma_{C}\right)$ is the first Betti number. Let $\Delta$ be a set of nodes of $C$, i.e., of edges of $\Gamma_{C}$. We denote by $\bar{\Delta}$ the subgraph of $\Gamma_{C}$ consisting of $\Delta$ plus the abutting vertices.

We say that a nodal curve $X$ is obtained from $C$ by blowing-up $\Delta$ if there exists $\pi: X \rightarrow C$ such that $\pi^{-1}\left(n_{i}\right)=E_{i} \simeq \mathbb{P}^{1}$ for any $n_{i} \in \Delta$, and $\pi: X \backslash \bigcup_{i} E_{i} \rightarrow C \backslash \Delta$ is an isomorphism. We call $\pi: X \rightarrow C$ a blow-up of $C$ and $E_{i}$ an exceptional component. Set $\widetilde{X}=\overline{X \backslash \bigcup_{i} E_{i}}$. Then $\widetilde{\pi}:=\pi_{\mid \widetilde{X}}: \widetilde{X} \rightarrow C$ is the normalization of $C$ at $\Delta$. For any $n_{i} \in \Delta$, set $\left\{p_{i}, q_{i}\right\}=E_{i} \cap \widetilde{X}=\widetilde{\pi}^{-1}\left(n_{i}\right)$; the points $p_{i}$ and $q_{i}$ are called exceptional nodes.

For families of curves, we use the same letters but different styles to denote the total space and a special fiber, and a star superscript to indicate restriction to the complement of the special fiber. For instance, $\mathcal{C} \rightarrow B$ will denote a family of curves with special fiber $C$, while $\mathcal{C}^{*}$ will stand for $\mathcal{C} \backslash C$.

Let $\mathcal{C} \rightarrow B$ be a family of nodal curves. We denote by $C_{b}$ the fiber over $b \in B$. We say that a family of nodal curves $\mathcal{X} \rightarrow B$, endowed with a $B$-morphism $\pi: \mathcal{X} \rightarrow \mathcal{C}$, is a family of blow-ups of $\mathcal{C}$, if for any $b \in B$ the restriction $\pi_{\mid X_{b}}: X_{b} \rightarrow C_{b}$ is a blow-up of $C_{b}$. If $\mathcal{N} \in \operatorname{Pic} \mathcal{C}$, we set $N_{b}:=\mathcal{N}_{\mid C_{b}}$ and, as above, $\mathcal{N}^{*}=\mathcal{N}_{\mid \mathcal{C}^{*}}$.

We denote by $S$ a smooth, connected 1-dimensional scheme, not necessarily complete, with a fixed point $s_{0} \in S$, and we set $S^{*}=S \backslash\left\{s_{0}\right\}$.

We shall attach to $X$ a second graph $\Sigma_{X}$, whose vertices are the connected components of $\widetilde{X}$ and whose edges are the exceptional components of $X$. Note that $\Sigma_{X}$ is obtained from $\Gamma_{C}$ by contracting all edges corresponding to nodes which are not blown up in $X$.

For any graph $\Gamma$ and commutative group $G$, we denote by $\mathcal{C}^{0}(\Gamma, G)$ and $\mathcal{C}^{1}(\Gamma, G)$ the groups of formal linear combinations, respectively, of vertices and edges of $\Gamma$ with coefficients in $G$. When we fix an orientation for $\Gamma$, the boundary and coboundary operators $\partial: \mathcal{C}^{1}(\Gamma, G) \rightarrow \mathcal{C}^{0}(\Gamma, G)$ and $\delta: \mathcal{C}^{0}(\Gamma, G) \rightarrow \mathcal{C}^{1}(\Gamma, G)$ are defined in the usual way. Then $H_{1}(\Gamma, G):=\operatorname{ker} \partial \subset \mathcal{C}^{1}(\Gamma, G)$.

For any positive integer $r, \mu_{r}$ is group of $r$-th roots of unity.

\section{Limit RoOtS OF LINE BUNDLES}

Let $C$ be a nodal curve, $r$ a positive integer, and $N$ a line bundle on $C$ whose degree is divisible by $r$.

\subsection{The main definition.}

Definition 2.1.1. Consider a triple $(X, L, \alpha)$, where $X$ is a blow-up of $C, L$ is a line bundle on $X$, and $\alpha$ is a homomorphism $\alpha: L^{\otimes r} \rightarrow \pi^{*}(N)(\pi: X \rightarrow C$ is the 
natural map). We say that $(X, L, \alpha)$ is a limit $r$-th root of $(C, N)$ if the following properties are satisfied:

(i) the restriction of $L$ to every exceptional component of $X$ has degree 1 ;

(ii) the map $\alpha$ is an isomorphism at all points of $X$ not belonging to an exceptional component;

(iii) for every exceptional component $E_{i}$ of $X$, the orders of vanishing of $\alpha$ at $p_{i}$ and $q_{i}$ add up to $r$.

The following is an analogue, for roots of line bundles, of the stable reduction theorem.

Proposition 2.1.2. Let $\mathcal{C} \rightarrow S$ be a family of nodal curves, smooth over $S^{*}$. Consider line bundles $\mathcal{N} \in \operatorname{Pic} \mathcal{C}$ and $\mathcal{L} \in \operatorname{Pic} \mathcal{C}^{*}$ with an isomorphism $\alpha: \mathcal{L}^{\otimes r} \stackrel{\sim}{\rightarrow}$ $\mathcal{N}_{\mid \mathcal{C}^{*}}$. Then there exist:

1) A diagram

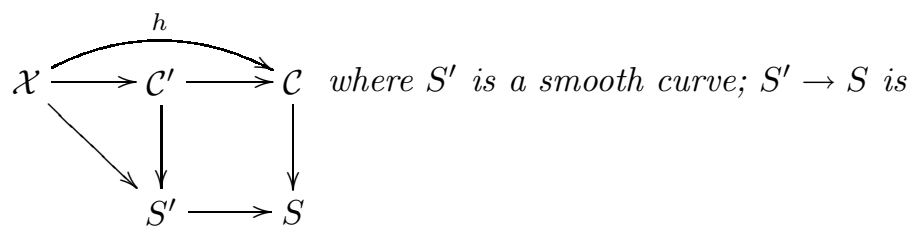

a finite morphism of degree $r$, étale over $S^{*} ; \mathcal{C}^{\prime}:=\mathcal{C} \times{ }_{S} S^{\prime} ; \mathcal{X} \rightarrow \mathcal{C}^{\prime}$ is an isomorphism outside the central fiber; $X$ is a blow-up of $C^{\prime}$.

2) A line bundle $\mathcal{L}^{\prime} \in \operatorname{Pic} \mathcal{X}$ such that $\mathcal{L}_{\mid \mathcal{X}^{*}}^{\prime}=h^{*}(\mathcal{L})$.

3) An isomorphism $\alpha^{\prime}:\left(\mathcal{L}^{\prime}\right)^{\otimes r} \stackrel{\sim}{\rightarrow} h^{*}(\mathcal{N})(-D)$, where $D$ is an effective Cartier divisor supported on the exceptional components of the central fiber, such that $\alpha_{\mid \mathcal{X}^{*}}^{\prime}=h^{*}(\alpha)$ and $\left(X, \mathcal{L}_{\mid X}^{\prime}, \alpha_{\mid X}^{\prime}\right)$ is a limit $r$-th root of $\left(C, \mathcal{N}_{\mid C}\right)$.

Moreover, for any $\left(\mathcal{X}_{1} \rightarrow \mathcal{C}^{\prime}, \mathcal{L}_{1}^{\prime}, \alpha_{1}^{\prime}, D_{1}\right)$ and $\left(\mathcal{X}_{2} \rightarrow \mathcal{C}^{\prime}, \mathcal{L}_{2}^{\prime}, \alpha_{2}^{\prime}, D_{2}\right)$ satisfying the above conditions, there exists an isomorphism $\sigma: \mathcal{X}_{1} \stackrel{\sim}{\rightarrow} \mathcal{X}_{2}$ over $\mathcal{C}^{\prime}$ such that $\sigma^{*}\left(D_{2}\right)=D_{1}, \sigma^{*}\left(\mathcal{L}_{2}^{\prime}\right) \simeq \mathcal{L}_{1}^{\prime}$, and this last isomorphism is compatible with $\alpha_{1}^{\prime}$ and $\alpha_{2}^{\prime}$.

The proof is in [14, section $\S 4.2 .2$. For more details on the work of Jarvis on the subject, see section 4.2 .

The notion of a limit root generalizes to families in a natural way. Let $\mathcal{C} \rightarrow T$ be a family of nodal curves and $\mathcal{N} \in \operatorname{Pic} \mathcal{C}$. A limit $r$-th root $(\mathcal{X}, \mathcal{L}, \alpha)$ of $(\mathcal{C}, \mathcal{N})$ is the datum of a family $\pi: \mathcal{X} \rightarrow \mathcal{C}$ of blow-ups of $\mathcal{C}$, a line bundle $\mathcal{L} \in$ Pic $\mathcal{X}$ and a homomorphism $\alpha: \mathcal{L}^{\otimes r} \rightarrow \pi^{*}(\mathcal{N})$, such that for all $t \in T,\left(X_{t}, L_{t}, \alpha_{t}\right)$ is a limit $r$-th root of $\left(C_{t}, N_{t}\right)$.

We can restate Proposition 2.1 .2 by saying that if $\mathcal{C} \rightarrow S$ is a family of nodal curves, $\mathcal{N} \in \operatorname{Pic} \mathcal{C}$ and $\left(\mathcal{C}^{*}, \mathcal{L}, \alpha\right)$ is an $r$-th root of $\mathcal{N}_{\mid \mathcal{C}^{*}}$, then for some finite covering $S^{\prime} \rightarrow S$ of degree $r$ there exists a limit $r$-th $\operatorname{root}\left(\mathcal{X}, \mathcal{L}^{\prime}, \alpha^{\prime}\right)$ of the pull-back of $\mathcal{N}$ to the base-changed family $\mathcal{C}^{\prime} \rightarrow S^{\prime}$ which extends over all of $S^{\prime}$ the pull-back of $\left(\mathcal{C}^{*} \rightarrow S^{*}, \mathcal{L}, \alpha\right)$.

2.2. Weighted graphs. To a limit $r$-th $\operatorname{root}(X, L, \alpha)$ we shall attach a weighted subgraph of the dual graph $\Gamma_{C}$ of $C$. The underlying graph is the subgraph $\bar{\Delta}$ corresponding to the set $\Delta$ of nodes which are blown up in $\pi: X \rightarrow C$. The weight function $w$ is defined on the exceptional nodes (or, equivalently, on the half-edges of $\bar{\Delta})$, takes values in $\{1, \ldots, r-1\}$, and is given by $w\left(p_{i}\right)=u_{i}, w\left(q_{i}\right)=v_{i}$, where 
$u_{i}, v_{i}$ are the orders of vanishing of $\alpha$ at the points $p_{i}, q_{i}$ respectively. We denote this weighted graph by $\Delta^{w}$. Clearly, the following conditions are satisfied:

(C1) $u_{i}+v_{i}=r$ for any $i$;

(C2) for every irreducible component $C_{j}$ of $C$, the sum of all weights assigned to the vertex corresponding $C_{j}$ is congruent to $\operatorname{deg}_{C_{j}} N$ modulo $r$.

Conversely, for any assignment of a weighted subgraph $\Delta^{w}$ of $\Gamma_{C}$ satisfying conditions $(\mathrm{C} 1)$ and $(\mathrm{C} 2)$, there is a limit $r$-th root of $N$ whose graph is $\Delta^{w}$. In fact, let $\widetilde{\pi}: \widetilde{X} \rightarrow C$ be the partial normalization of $C$ at $\Delta$. By (C2), the line bundle $\widetilde{\pi}^{*}(N)\left(-\sum_{n_{i} \in \Delta}\left(u_{i} p_{i}+v_{i} q_{i}\right)\right)$ on $\widetilde{X}$ admits an $r$-th $\operatorname{root} \widetilde{L} \in \operatorname{Pic} \widetilde{X}$. Let $X$ be the blow-up of $C$ at $\Delta$. We obtain a limit $\operatorname{root}(X, L, \alpha)$ of $N$ by gluing to $\widetilde{L}$ (however we wish) the degree one line bundle on each exceptional component; the map $\alpha: L^{\otimes r} \rightarrow \pi^{*}(N)$ is defined to agree with $(\widetilde{L})^{\otimes r}=\widetilde{\pi}^{*}(N)\left(-\sum_{i}\left(u_{i} p_{i}+v_{i} q_{i}\right)\right) \hookrightarrow$ $\widetilde{\pi}^{*}(N)$ on $\widetilde{X}$, and to be zero on all exceptional components.

Remark 2.2.1. The multidegree $\underline{d}=\underline{\operatorname{deg}} N$ of $N$ is the set of the degrees $\operatorname{deg}_{C_{j}} N$ for all irreducible components $C_{j}$ of $C$. Its class modulo $r$ can be viewed as an element $[\underline{d}]_{r}$ of $\mathcal{C}^{0}\left(\Gamma_{C}, \mathbb{Z} / r\right)$ defined naturally as

$$
[\underline{d}]_{r}:=\sum_{j}\left(\operatorname{deg}_{C_{j}} N \quad(\bmod r)\right)\left[C_{j}\right] .
$$

Of course, condition (C2) above only depends on $[\underline{d}]_{r}$.

Now fix an orientation for $\Gamma_{C}$; then there is a natural bijection between $\mathcal{C}^{1}\left(\Gamma_{C}, \mathbb{Z} / r\right)$ and the set of weighted subgraphs of $\Gamma_{C}$ satisfying $(\mathrm{C} 1)$ : to a weighted subgraph $\Delta^{w}$ we associate the 1-chain $\sum_{i} u_{i}\left[n_{i}\right]$ with the convention that the edge $n_{i}$ is oriented entering the vertex corresponding to $u_{i}$.

In this set-up, the boundary operator $\partial: \mathcal{C}^{1}\left(\Gamma_{C}, \mathbb{Z} / r\right) \rightarrow \mathcal{C}^{0}\left(\Gamma_{C}, \mathbb{Z} / r\right)$ can be viewed as a map from weighted subgraphs of $\Gamma_{C}$ satisfying $(\mathrm{C} 1)$ to multidegrees reduced modulo $r$. Then $\Delta^{w}$ satisfies (C2) with respect to $[\underline{d}]_{r}$ if and only if $\partial\left(\Delta^{w}\right)=$ $[\underline{d}]_{r}$. Since the cardinality of $\partial^{-1}\left([\underline{d}]_{r}\right)$ equals that of $\operatorname{ker} \partial=H_{1}\left(\Gamma_{C}, \mathbb{Z} / r\right)$, we see that:

- for every $N$ as above, there exist $r^{b_{1}\left(\Gamma_{C}\right)}$ weighted subgraphs of $\Gamma_{C}$ satisfying (C1) and (C2) with respect to $N$.

\subsection{Automorphisms of limit roots.}

Definition 2.3.1. An isomorphism of limit $r$-th $\operatorname{roots}(\mathcal{X} \rightarrow T, \mathcal{L}, \alpha)$ and $\left(\mathcal{X}^{\prime} \rightarrow\right.$ $\left.T, \mathcal{L}^{\prime}, \alpha^{\prime}\right)$ of $(\mathcal{C}, \mathcal{N})$ is the datum of:

(a) an isomorphism $\sigma: \mathcal{X} \rightarrow \mathcal{X}^{\prime}$ over $\mathcal{C}$,

(b) an isomorphism $\tau: \sigma^{*} \mathcal{L}^{\prime} \rightarrow \mathcal{L}$ that makes the following diagram commute:

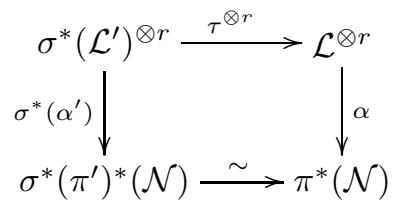

This notion of isomorphism agrees with the one of 9, rather than with the one of 8 . At the level of automorphisms, for instance, the difference is that here fiber multiplication in $\mathcal{L}$ by a nontrivial root of unity is viewed as a nontrivial automorphism of the limit root. To make contact with the terminology of [8] and 
9], an isomorphism between $\mathcal{X}$ and $\mathcal{X}^{\prime}$ over $\mathcal{C}$ will sometimes be referred to as an inessential isomorphism. Likewise, automorphisms of $\mathcal{X}$ over $\mathcal{C}$ will sometimes be called inessential automorphisms of $\mathcal{X}$. As customary, the group of these automorphism will be denoted $\operatorname{Aut}_{\mathcal{C}}(\mathcal{X})$. Given a limit root $(\mathcal{X} \rightarrow T, \mathcal{L}, \alpha)$, we denote by $\operatorname{Aut}(\mathcal{X} \rightarrow T, \mathcal{L}, \alpha)$ the group of its automorphisms. Clearly, $\operatorname{Aut}(\mathcal{X} \rightarrow T, \mathcal{L}, \alpha)$ maps to $\operatorname{Aut}_{\mathcal{C}}(\mathcal{X})$; the kernel is naturally isomorphic to a product of copies of $\mu_{r}$, one for each connected component of $T$.

We now state and prove some of the basic properties of automorphisms of limit roots. Let $X$ be a blow-up of $C$, and let $E_{1}, \ldots, E_{m}$ be its exceptional components. View each $E_{i}$ as a copy of the Riemann sphere, with the origin placed at $q_{i}$ and the point at infinity placed at $p_{i}$. Then any inessential automorphism of $X$ acts on each $E_{i}$ as multiplication by a nonzero constant $l_{i}$, and any assignment of the $l_{i}$ corresponds to an inessential automorphism. This proves the first part of the following result.

Lemma 2.3.2. Let $(X, L, \alpha)$ be a limit root of $(C, N)$, and fix an orientation on the graph $\Sigma_{X}$. Then:

(i) There is a natural identification $\operatorname{Aut}_{C}(X) \simeq \mathcal{C}^{1}\left(\Sigma_{X}, \mathbb{C}^{*}\right)$.

(ii) There is a natural identification $\operatorname{Aut}(X, L, \alpha) \simeq \mathcal{C}^{0}\left(\Sigma_{X}, \mu_{r}\right)$, and the homomorphism $\operatorname{Aut}(X, L, \alpha) \rightarrow \operatorname{Aut}_{C}(X)$ corresponds to the composition of the coboundary map $\delta: \mathcal{C}^{0}\left(\Sigma_{X}, \mu_{r}\right) \rightarrow \mathcal{C}^{1}\left(\Sigma_{X}, \mu_{r}\right)$ with the inclusion $\mathcal{C}^{1}\left(\Sigma_{X}, \mu_{r}\right) \rightarrow \mathcal{C}^{1}\left(\Sigma_{X}, \mathbb{C}^{*}\right)$

Proof. We have already proved (i). To prove (ii), it is convenient to work with the geometric bundles $\mathbb{V}(L)$ and $\mathbb{V}\left(L^{\otimes r}\right)$ rather than with $L$ and $L^{\otimes r}$. In terms of these, an automorphism of $(X, L, \alpha)$ is a pair $(\sigma, \tau)$, where $\sigma$ is an inessential automorphism of $X$ and $\tau$ is an automorphism of $\mathbb{V}(L)$ which is compatible with $\sigma$, is linear on the fibers of $\mathbb{V}(L) \rightarrow X$, and is such that $\alpha \circ \tau^{\otimes r}=\alpha$. This last condition means that, above each connected component $X_{j}$ of $\tilde{X}, \tau^{\otimes r}$ is the identity, and hence $\tau$ must be the fiberwise multiplication by an $r$-th root of unity $l_{j}$. If $E_{i}$ is an exceptional component connecting $X_{j}$ and $X_{k}$, the restrictions of $\tau$ and $\sigma$ to $\mathbb{V}(L)_{\mid E_{i}} \rightarrow E_{i}$ can thus be viewed as an automorphism pair of $\mathbb{V}(\mathcal{O}(1)) \rightarrow \mathbb{P}^{1}$ which is the multiplication by $l_{j}$ on the fiber above the origin and the multiplication by $l_{k}$ on the fiber above the point at infinity. These pairs are easy to describe. View $\mathbb{P}^{1}$ as the projective space $\mathbb{P} V$ constructed on a two-dimensional vector space $V$, and its origin and point at infinity as $\left[v_{0}\right]$ and $\left[v_{1}\right]$, where $v_{0}, v_{1}$ is a basis of $V$. Then $\mathbb{V}(\mathcal{O}(1))$ is the set of all pairs $(\ell, \varphi)$, where $\ell$ is a one-dimensional subspace of $V$ and $\varphi$ an element of its dual. An automorphism pair as above then acts on $\mathbb{V}(\mathcal{O}(1))$ via $(\ell, \varphi) \mapsto\left(f(\ell), \varphi \circ f^{-1}\right)$, where $f$ is the automorphism of $V$ such that $f\left(v_{0}\right)=l_{j}^{-1} v_{0}$, $f\left(v_{1}\right)=l_{k}^{-1} v_{0}$, and on $\mathbb{P} V$ via $\ell \mapsto f(\ell)$. Thus $\sigma$ acts on $E_{i}$ as multiplication by $l_{j} / l_{k}$. This means that giving an automorphism of $(X, L, \alpha)$ is the same as giving an $r$-th root of unity for each connected component of $\widetilde{X}$, that is, an element of $\mathcal{C}^{0}\left(\Sigma_{X}, \mu_{r}\right)$, and that the map $\operatorname{Aut}(X, L, \alpha) \rightarrow \operatorname{Aut}_{C}(X)$ is as claimed.

It follows from Lemma 2.3.2 that $\operatorname{Aut}(X, L, \alpha)$ has cardinality $r^{\gamma}$, where $\gamma$ is the number of connected components of $\widetilde{X}$.

Fix $(C, N)$ and $\pi: X \rightarrow C$ as usual. We need to further investigate the action of $\operatorname{Aut}_{C}(X)$ on certain line bundles on $X$. Consider pairs $(M, \beta)$, where $M$ is a line bundle on $X, \beta: M \rightarrow \pi^{*}(N)$ is a homomorphism which is an isomorphism on 
the complement of the exceptional components of $X$, and $M$ is assumed to have degree $r$ on each exceptional component. This degree condition forces $\beta$ to vanish identically on each exceptional component

An example of this situation is provided by $\alpha: L^{\otimes r} \rightarrow \pi^{*}(N)$, where $(X, L, \alpha)$ is a limit $r$-th root.

Lemma 2.3.3. Let $(M, \beta)$ and $\left(M^{\prime}, \beta^{\prime}\right)$ be two pairs as above. Then:

(i) There exist $\sigma \in \operatorname{Aut}_{C}(X)$ and an isomorphism $\vartheta: M \rightarrow \sigma^{*}\left(M^{\prime}\right)$ such that $\beta=\sigma^{*}\left(\beta^{\prime}\right) \circ \vartheta$ if and only if, for any exceptional node $q$, the orders of vanishing of $\beta$ and $\beta^{\prime}$ at $q$ are the same.

(ii) Under the identification (i) of Lemma 2.3.2, the set of those $\sigma \in \operatorname{Aut}_{C}(X)$ such that there is an isomorphism $\vartheta: M \rightarrow \sigma^{*}(M)$ with the property that $\beta=\sigma^{*}(\beta) \circ \vartheta$ corresponds to $\mathcal{C}^{1}\left(\Sigma_{X}, \mu_{r}\right)$.

Proof. The proof is similar to the one of Lemma 2.3.2. The "only if" part of (i) is clear. To prove the converse, denote by $u_{i}$ (resp., $v_{i}$ ) the order of vanishing of $\beta$ and $\beta^{\prime}$ at $p_{i}$ (resp., $q_{i}$ ). The homomorphisms $\beta$ and $\beta^{\prime}$ determine isomorphisms $M_{\mid \widetilde{X}} \simeq$ $\pi^{*}(N)\left(-\sum\left(u_{i} p_{i}+v_{i} q_{i}\right)\right) \simeq M_{\mid \widetilde{X}}^{\prime}$, hence there is a unique isomorphism $\vartheta^{\prime}: M_{\mid \widetilde{X}} \rightarrow$ $M_{\mid \widetilde{X}}^{\prime}$ such that $\beta_{\mid \widetilde{X}}=\beta_{\mid \widetilde{X}}^{\prime} \circ \vartheta^{\prime}$. Clearly, $\vartheta$ must restrict to $\vartheta^{\prime}$ on $\widetilde{X}$. It remains to construct $\sigma$ and $\vartheta$ on the exceptional components. If $E_{i}$ is one of these, we identify the restrictions to $E_{i}$ of $\mathbb{V}(M) \rightarrow X$ and $\mathbb{V}\left(M^{\prime}\right) \rightarrow X$ to $\mathbb{V}(\mathcal{O}(r)) \rightarrow \mathbb{P}(V)$, where $V$ is a two-dimensional vector space. We also think of $\mathbb{P}(V)$ as being attached to the rest of $X$ at $\left[v_{0}\right]$ and $\left[v_{1}\right]$, where $v_{0}, v_{1}$ is a basis of $V$. The isomorphisms $\vartheta$ and $\sigma$, if they exist, restrict on $\mathbb{V}(\mathcal{O}(r)) \rightarrow \mathbb{P}(V)$ to an automorphism pair fixing $\left[v_{0}\right]$ and $\left[v_{1}\right]$. Now, $\mathbb{V}(\mathcal{O}(r))$ is the set of all pairs $\left(\ell, \varphi^{\otimes r}\right)$, where $\ell$ is a one-dimensional subspace of $V$ and $\varphi$ an element of its dual. Any automorphism pair fixing $\left[v_{0}\right]$ and $\left[v_{1}\right]$ then acts on $\mathbb{V}(\mathcal{O}(r))$ via $\left(\ell, \varphi^{\otimes r}\right) \mapsto\left(f(\ell),\left(\varphi \circ f^{-1}\right)^{\otimes r}\right)$, where $f$ is an automorphism of $V$ such that $f\left(v_{0}\right)=b_{0} v_{0}, f\left(v_{1}\right)=b_{1} v_{0}, b_{0}, b_{1} \in \mathbb{C}^{*}$, and on $\mathbb{P} V$ via $\ell \mapsto f(\ell)$. The action of such an automorphism pair on the fiber above $\left[v_{i}\right], i=0,1$, corresponds to multiplication by $b_{i}^{-r}$, hence we can choose $b_{0}$ and $b_{1}$ so that it matches the one of $\vartheta^{\prime}$; the choices are unique up to multiplication by $r$-th roots of unity. This proves the existence of $\sigma$ and $\vartheta$. It also proves (ii). In fact, $\sigma$ acts on $E_{i}$ as multiplication by $b_{1} / b_{0}$. In the setup of (ii), both $b_{0}$ and $b_{1}$ are (arbitrary) roots of unity. The conclusion is that $\sigma$ acts on each exceptional component as multiplication by an $r$-th root of unity, and conversely that any element of $\mathcal{C}^{1}\left(\Sigma_{X}, \mu_{r}\right)$ comes from an automorphism $\sigma$ as in (ii).

2.4. The general moduli problem. Let $B$ be a scheme, $f: \mathcal{C} \rightarrow B$ a family of nodal curves, and $\mathcal{N} \in$ Pic $\mathcal{C}$ a line bundle of relative degree divisible by $r$. We shall use the following notation: for any $B$-scheme $T$ with structure map $p: T \rightarrow B$ we set

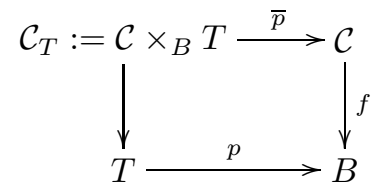

where $\bar{p}: \mathcal{C}_{T} \rightarrow \mathcal{C}$ is the projection. The pull-back of $\mathcal{N}$ to $\mathcal{C}_{T}$ is denoted by

$$
\mathcal{N}_{T}:=\bar{p}^{*} \mathcal{N} \text {. }
$$


Having fixed $B, f: \mathcal{C} \rightarrow B$ and $\mathcal{N}$ as above, we introduce a contravariant functor

$$
\overline{\mathcal{S}}_{f}^{r}(\mathcal{N}):\{B \text {-schemes }\} \longrightarrow\{\text { sets }\}
$$

encoding the moduli problem for limit $r$-th roots of $\mathcal{N}$, as follows. For every $B$ scheme $T$, the set $\overline{\mathcal{S}}_{f}^{r}(\mathcal{N})(T)$ is the set of all limit $r$-th roots of $\mathcal{N}_{T}$ (which is, with the above notation, a line bundle on the total space of the family $\mathcal{C}_{T} \rightarrow T$ ), modulo isomorphisms of limit roots.

For every morphism of $B$-schemes $\rho: T \rightarrow T^{\prime}$, the corresponding map

$$
\overline{\mathcal{S}}_{f}^{r}(\mathcal{N})(\rho): \overline{\mathcal{S}}_{f}^{r}(\mathcal{N})\left(T^{\prime}\right) \longrightarrow \overline{\mathcal{S}}_{f}^{r}(\mathcal{N})(T)
$$

sends a limit $r$-th $\operatorname{root}\left(\mathcal{X}^{\prime}, \mathcal{L}^{\prime}, \alpha^{\prime}\right)$ of $\mathcal{N}_{T^{\prime}}$ to the limit root $(\mathcal{X}, \mathcal{L}, \alpha)$ of $\mathcal{N}_{T}$ defined by $\mathcal{X}:=\mathcal{X}^{\prime} \times_{T^{\prime}} T$ and $\mathcal{L}:=\bar{\rho}^{*} \mathcal{L}^{\prime}$, where $\bar{\rho}: \mathcal{X} \rightarrow \mathcal{X}^{\prime}$ is the projection. Finally, the morphism $\alpha^{\prime}$ is the natural pull-back of $\alpha$.

We shall prove

Theorem 2.4.1. Let $f: \mathcal{C} \rightarrow B$ be a family of nodal curves, with $B$ a quasiprojective scheme. Let $\mathcal{N} \in \operatorname{Pic} \mathcal{C}$ be a line bundle on $\mathcal{C}$ of relative degree divisible by $r$.

Then the functor $\overline{\mathcal{S}}_{f}^{r}(\mathcal{N})$ is coarsely represented by a quasiprojective scheme $\bar{S}_{f}^{r}(\mathcal{N})$, finite over $B$. If $B$ is projective, then $\bar{S}_{f}^{r}(\mathcal{N})$ is projective.

We shall denote by $S_{f}^{r}(\mathcal{N})$ the open subscheme of $\bar{S}_{f}^{r}(\mathcal{N})$ parametrizing (closed) points whose underlying curve has no exceptional components.

Proposition 2.4.2. Let $\xi=(X, L, \alpha) \in \bar{S}_{f}^{r}(\mathcal{N})$. The morphism $p: \bar{S}_{f}^{r}(\mathcal{N}) \rightarrow B$ is smooth at $\xi$ if and only if $b_{1}\left(\Sigma_{X}\right)=0$.

In particular, if $B$ is smooth, $\bar{S}_{f}^{r}(\mathcal{N})$ is smooth at all points $\xi$ such that $X$ has no exceptional components, or is of compact type.

The proofs of these results are in section 3.4 .

Remark 2.4.3. A purely formal consequence of Theorem 2.4.1 is the following useful fact. Let $B^{\prime}$ be a $B$-scheme, and denote by

$$
f^{\prime}: \mathcal{C}^{\prime}=\mathcal{C} \times{ }_{B} B^{\prime} \longrightarrow B^{\prime}
$$

the family obtained by base change, and by $\mathcal{N}^{\prime}$ the pull-back of $\mathcal{N}$ to $\mathcal{C}^{\prime}$. Then we naturally have

$$
\bar{S}_{f^{\prime}}^{r}\left(\mathcal{N}^{\prime}\right) \simeq \bar{S}_{f}^{r}(\mathcal{N}) \times_{B} B^{\prime}
$$

Remark 2.4.4. Let $f: \mathcal{C} \rightarrow B$ and $\mathcal{N} \in \operatorname{Pic} \mathcal{C}$ be as above. For any $\mathcal{L}_{0} \in \operatorname{Pic} \mathcal{C}$, the functors $\overline{\mathcal{S}}_{f}^{r}(\mathcal{N})$ and $\overline{\mathcal{S}}_{f}^{r}\left(\mathcal{N} \otimes \mathcal{L}_{0}^{\otimes r}\right)$ are isomorphic, so the same holds for the corresponding moduli spaces $\bar{S}_{f}^{r}(\mathcal{N})$ and $\bar{S}_{f}^{r}\left(\mathcal{N} \otimes \mathcal{L}_{0}^{\otimes r}\right)$. This is because for any limit $r$-th $\operatorname{root}(\mathcal{X}, \mathcal{L})$ of $\mathcal{N},\left(\mathcal{X}, \mathcal{L} \otimes \pi^{*}\left(\mathcal{L}_{0}\right)\right)$ is a limit $r$-th root of $\mathcal{N} \otimes \mathcal{L}_{0}^{\otimes r}$.

Remark 2.4.5. Let $f: \mathcal{C} \rightarrow B$ and $\mathcal{N} \in$ Pic $\mathcal{C}$ be as above. For any positive integer $s$, there is a natural injective morphism

$$
J: \bar{S}_{f}^{r}(\mathcal{N}) \longrightarrow \bar{S}_{f}^{r s}\left(\mathcal{N}^{\otimes s}\right)
$$

over $B$. Indeed for any limit $r$-th $\operatorname{root}(\mathcal{X}, \mathcal{L}, \alpha)$ of $\mathcal{N},\left(\mathcal{X}, \mathcal{L}, \alpha^{\otimes s}\right)$ is a limit $(r s)$-th root of $\mathcal{N}^{\otimes s}$. The image of $J$ is a union of irreducible components of $\bar{S}_{f}^{r s}\left(\mathcal{N}^{\otimes s}\right)$. 


\section{Proof of the Existence theorem}

We shall now construct $\bar{S}_{f}^{r}(\mathcal{N})$, and prove Theorem 2.4.1. First of all, working in the analytic category, we construct the universal deformation of a limit root. Then we glue together the bases of the universal deformations, in order to give a complex algebraic structure to $\bar{S}_{f}^{r}(\mathcal{N})$.

We will make use of the following simple results. The first one is well known, while the second is Lemma 1.1 of 8 .

Remark 3.0.6. Let $\mathcal{Y} \rightarrow T$ be a family of nodal curves, $t_{0}$ a point of $T$, and let $Y$ be the fiber over $t_{0}$. Consider two line bundles $\mathcal{P} \in \operatorname{Pic} \mathcal{Y}$ and $L \in \operatorname{Pic} Y$ such that there exists an isomorphism $\iota_{0}: L^{\otimes r} \rightarrow \mathcal{P}_{\mid Y}$. Then, up to shrinking $T$, there exist a line bundle $\mathcal{L} \in \operatorname{Pic} \mathcal{Y}$ extending $L$ and an isomorphism $\iota: \mathcal{L}^{\otimes r} \rightarrow \mathcal{P}$ extending $\iota_{0}$. Moreover, this extension is unique up to isomorphism, meaning that if $\left(\mathcal{L}^{\prime}, \iota^{\prime}\right)$ is another extension, then there is an isomorphism $\chi: \mathcal{L} \rightarrow \mathcal{L}^{\prime}$ on a neighbourhood of $Y$ which restricts to the identity on $L$ and is such that $\iota=\iota^{\prime} \circ \chi^{\otimes r}$.

Remark 3.0.7. Let $\mathcal{Y} \rightarrow T$ be a family of nodal curves, and let $E$ be a smooth rational component of one of its fibers. If $\mathcal{L}$ is a line bundle on $\mathcal{Y}$ whose restriction to $E$ has zero degree, then $\mathcal{L}$ is trivial on a neighbourhood of $E$ in $\mathcal{Y}$.

3.1. Local structure near exceptional curves. Let $\mathcal{C} \rightarrow T$ be a family of nodal curves, $\mathcal{N} \in \operatorname{Pic} \mathcal{C}$ and $(\mathcal{X} \rightarrow T, \mathcal{L}, \alpha)$ a limit $r$-th root of $(\mathcal{C}, \mathcal{N})$. Fix an exceptional component $E \subset \mathcal{X}$ lying over a point $t_{0} \in T$, let $u, v$ be the corresponding weights, and denote by $n$ the point of $\mathcal{C}$ to which $E$ contracts. As usual, $\pi: \mathcal{X} \rightarrow \mathcal{C}$ is the natural map; recall that $\pi^{*} \mathcal{N}$ is trivial on a neighbourhood of $E$. We shall describe the geometry of $\mathcal{X} \rightarrow T$ near $E$.

The prototype is the following family: for the base, consider the affine plane curve $C_{u, v} \subset \mathbb{A}_{w, 2}^{2}, 3$ given by the equation $w^{u}=z^{v}$. The total space is the surface

$$
S_{u, v}=\left\{x s_{0}=w s_{1}, y s_{1}=z s_{0}, w^{u}=z^{v}\right\} \subset \mathbb{A}_{x, y, w, z}^{4} \times \mathbb{P}_{s_{0}: s_{1}}^{1}
$$

The projection to $\mathbb{A}_{w, z}^{2}$ yields a family of open curves

$$
f_{u, v}: S_{u, v} \longrightarrow C_{u, v}
$$

whose only singular fiber lies over the point $(0,0) \in C_{u, v}$ and contains an exceptional component, i.e., the locus of all points of $S_{u, v}$ for which $x=y=w=z=0$.

Note that $S_{u, v}$ has $d=(u, v)$ irreducible components, each one isomorphic to $S_{u / d, v / d}$. For instance, when $d=1, S_{u, v}$ is smooth if and only if $u=v=1$ (in which case it is a resolution of an $A_{1}$-singularity); it is normal if and only if $u=1$ or $v=1$ (in which case it has an isolated $A_{v-1}$ or $A_{u-1}$ singularity in one exceptional node). If $u>1$ and $v>1, S_{u, v}$ is singular along the whole special fiber of $f_{u, v}$ (the curve $w=z=0$ ).

We now show that the morphism $f_{u, v}: S_{u, v} \rightarrow C_{u, v}$ is a model for $\mathcal{X} \rightarrow T$ near $E$. To set up, recall that a neighbourhood $V$ of $n$ in $\mathcal{C}$ is of the form $\{x y=\ell\}$, where $\ell$ is a regular function on a neighbourhood $T_{0}$ of $t_{0}$. In other terms, there is

\footnotetext{
${ }^{3}$ The notation $\mathbb{A}_{w, z}^{2}$ means, here and similarly below, that $w, z$ are the coordinate functions on $\mathbb{A}^{2}$.
} 
a fiber product diagram

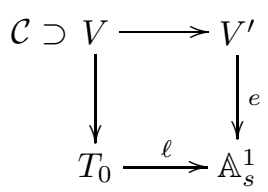

where $V^{\prime}$ is a neighbourhood of the origin in $\mathbb{A}_{x, y}^{2}$ and $e$ is given by $s=x y$.

The curve $C_{u, v}$ maps to $\mathbb{A}_{s}^{1}$ via $(w, z) \mapsto w z$, and $S_{u, v}$ to $\mathbb{A}_{x, y}^{2}$ via projection. We will show that $\ell$ lifts to a morphism $h: T_{0} \rightarrow C_{u, v}$. More precisely, we shall prove the following result.

Lemma 3.1.1. Possibly after shrinking $T_{0}$, diagram (11) lifts to a fiber product diagram

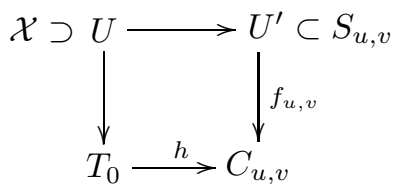

where $U$ is a neighbourhood of $E$ in $\mathcal{X}$ and $U^{\prime}$ is a neighbourhood of the exceptional curve in $S_{u, v}$. Moreover, the lifting can be chosen so that the following holds. Let $\zeta_{\mathcal{N}}$ be any generator of $\pi^{*} \mathcal{N}$ over $U$, and denote by $U_{0}$ (resp., $U_{1}$ ) the complement in $U$ of the inverse image of the locus $s_{1}=0$ (resp., $\left.s_{0}=0\right)$. Then there are generators $\zeta_{0}$ and $\zeta_{1}$ of $\mathcal{L}^{\otimes r}$ over $U_{0}$ and $U_{1}$ such that

$$
s_{0}^{r} \zeta_{0}=s_{1}^{r} \zeta_{1} \text { on } U_{0} \cap U_{1}, \quad \alpha\left(\zeta_{0}\right)=x^{u} \zeta_{\mathcal{N}} \text { on } U_{0}, \quad \alpha\left(\zeta_{1}\right)=y^{v} \zeta_{\mathcal{N}} \text { on } U_{1} 4
$$

Proof. A weaker lifting property than the one claimed obviously holds. Consider the threefold

$$
Z=\left\{x s_{0}=w s_{1}, y s_{1}=z s_{0}\right\} \subset \mathbb{A}_{x, y, w, z}^{4} \times \mathbb{P}_{s_{0}: s_{1}}^{1} .
$$

The projection to $\mathbb{A}_{w, z}^{2}$ yields a family of open curves $\phi: Z \longrightarrow \mathbb{A}_{w, z}^{2}$, whose restriction to $C_{u, v}$ is clearly just $S_{u, v} \rightarrow C_{u, v}$. Then, after suitable shrinkings, diagram (11) lifts (nonuniquely) to

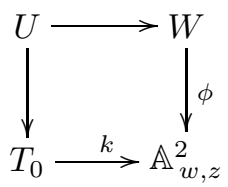

where $W \subset Z$ is a neighbourhood of the exceptional curve in the central fiber of $\phi$. In concrete terms, this means that $U$ has equations

$$
x \eta_{0}=k_{0} \text { in } U_{0}, \quad y \eta_{1}=k_{1} \text { in } U_{1},
$$

where $\eta_{0} \eta_{1}=1$ on $U_{0} \cap U_{1}, x$ and $y$ vanish on $E$, $\eta_{0}$ and $\eta_{1}$ vanish on $\widetilde{X} \cap U$, and $k_{0}$ and $k_{1}$ are regular functions on $T_{0}$ which vanish at $t_{0}$. Clearly, $\eta_{0}$ is the pull-back of the quotient $s_{0} / s_{1}$, and $\eta_{1}$ is its inverse. What must be shown is that $k$ can be chosen in such a way that $k_{0}^{u}=k_{1}^{v}$. This is where the presence of a limit root comes into play.

Consider the line bundle $\mathcal{M}$ on $U$ whose transition function, relative to the cover $\left\{U_{0}, U_{1}\right\}$, is $\eta_{1}^{r} \in \mathcal{O}^{*}\left(U_{0} \cap U_{1}\right)$. Clearly, $\operatorname{deg} \mathcal{M}_{\mid E}=\operatorname{deg} \mathcal{L}_{\mid E}^{\otimes r}=r$. By Remark

\footnotetext{
${ }^{4}$ For simplicity, we continue to use $x$ and $y$ to denote the pull-backs of these functions to $U$.
} 
3.0.7 we may assume that $\mathcal{L}_{\mid U}^{\otimes r} \simeq \mathcal{M}$, possibly after shrinking $U$. Thus there are generators $\zeta_{0}^{\prime}, \zeta_{1}^{\prime}$ of $\mathcal{L}^{\otimes r}$, over $U_{0}$ and $U_{1}$, respectively, such that $\zeta_{0}^{\prime}=\eta_{1}^{r} \zeta_{1}^{\prime}$ on $U_{0} \cap U_{1}$.

Let $\zeta_{\mathcal{N}}$ be a generator of $\pi^{*}(\mathcal{N})$ over $U$. We have $\alpha\left(\zeta_{i}^{\prime}\right)=c_{i} \zeta_{\mathcal{N}}$ on $U_{i}, i=0,1$, where $c_{0}$ and $c_{1}$ are regular functions on $U_{0}$ and $U_{1}$, respectively. Consider the expressions of $c_{0}$ and $c_{1}$ as power series

$$
c_{0}=\sum_{i \geq 0} a_{i} x^{i}+\sum_{i<0} a_{i} \eta_{0}^{-i} \text { in } U_{0}, \quad c_{1}=\sum_{i \geq 0} b_{i} y^{i}+\sum_{i<0} b_{i} \eta_{1}^{-i} \text { in } U_{1},
$$

where the $a_{i}$ and the $b_{i}$ are regular functions on $T_{0}$. Since $\pi^{*}(\mathcal{N})$ has degree zero on $E$ while $\mathcal{L}^{\otimes r}$ has degree $r, \alpha$ is identically zero on $E$, which gives $a_{i}\left(t_{0}\right)=b_{i}\left(t_{0}\right)=0$ for all $i \leq 0$. On the other hand, by definition, the order of vanishing of $\left(c_{0}\right)_{\mid X \cap U_{0}}$ at $p$ is $u$ and that of $\left(c_{1}\right)_{\mid X \cap U_{1}}$ at $q$ is $v$, so $a_{u}\left(t_{0}\right) \neq 0$ and $b_{v}\left(t_{0}\right) \neq 0$. Up to shrinking $T_{0}$, we may thus assume that $a_{u}$ and $b_{v}$ are units.

On $U_{0} \cap U_{1}$ we have $\alpha\left(\zeta_{0}^{\prime}\right)=\eta_{1}^{r} \alpha\left(\zeta_{1}^{\prime}\right)$, so $c_{0}=\eta_{1}^{r} c_{1}$. We also have $\eta_{0}=\eta_{1}^{-1}$, $x=k_{0} \eta_{1}, y=k_{1} \eta_{1}^{-1}$. Substituting, we get

$$
\sum_{i \geq 0} a_{i} k_{0}^{i} \eta_{1}^{i}+\sum_{i<0} a_{i} \eta_{1}^{i}=\sum_{i \geq 0} b_{i} k_{1}^{i} \eta_{1}^{r-i}+\sum_{i<0} b_{i} \eta_{1}^{r-i} \text { in } U_{0} \cap U_{1} .
$$

Comparing terms of degree $u$ in $\eta_{1}$, we get $a_{u} k_{0}^{u}=b_{v} k_{1}^{v}$. This is not exactly what we are asking for, but we may proceed as follows. Let $\delta$ be an $r$-th root of $b_{v} / a_{u}$ (a unit), and define a morphism $h: T_{0} \rightarrow \mathbb{A}_{w, z}^{2}$ by setting $h_{0}=\delta^{-1} k_{0}, h_{1}=\delta k_{1}$. Clearly, $h_{0}^{u}=h_{1}^{v}$, and $h$ is a lifting of $\ell$, since $h_{0} h_{1}=k_{0} k_{1}=\ell$. A compatible lifting of $V \rightarrow \mathbb{A}_{x, y}^{2}$ to a morphism $U \rightarrow S_{u, v}$ is given by

$$
\begin{aligned}
\left(x, \eta_{0}, t\right) & \mapsto\left(x, \eta_{0} k_{1}(t), h_{0}(t), h_{1}(t),\left[\delta^{-1} \eta_{0}: 1\right]\right) \quad \text { on } U_{0}, \\
\left(y, \eta_{1}, t\right) & \mapsto\left(\eta_{1} k_{0}(t), y, h_{0}(t), h_{1}(t),\left[1: \delta \eta_{1}\right]\right) \quad \text { on } U_{1},
\end{aligned}
$$

where $t$ stands for a variable point in $T_{0}$.

It remains to find the local generators $\zeta_{0}$ and $\zeta_{1}$. What we have done so far amounts to showing that, replacing $\eta_{0}$ with $\delta^{-1} \eta_{0}$ and $\eta_{1}$ with $\delta \eta_{1}$, we may assume that $U$ is defined by equations $x \eta_{0}=h_{0}$ on $U_{0}$ and $y \eta_{1}=h_{1}$ on $U_{1}$, where $\eta_{0} \eta_{1}=1$ on $U_{0} \cap U_{1}$ and $h_{0}^{u}=h_{1}^{v}$. Moreover, replacing $\zeta_{0}^{\prime}$ with $\delta^{u} \zeta_{0}^{\prime}$ and $\zeta_{1}^{\prime}$ with $\delta^{-v} \zeta_{1}^{\prime}$, we may also assume that the coefficients $a_{u}$ and $b_{v}$ in the power series developments (2) are equal, while conserving the property that $\zeta_{0}^{\prime}=\eta_{1}^{r} \zeta_{1}^{\prime}$. Now, comparing terms of all degrees in identity (3), we conclude that

$$
c_{0}=x^{u} \rho, \quad c_{1}=y^{v} \rho,
$$

where

$$
\rho=a_{u}+\sum_{i>0} a_{u+i} x^{i}+\sum_{i>0} b_{v+i} y^{i}=b_{v}+\sum_{i>0} a_{u+i} x^{i}+\sum_{i>0} b_{v+i} y^{i} .
$$

Since $\rho$ is a unit on a neighbourhood of $E$, possibly after shrinking $U$ it makes sense to set

$$
\zeta_{i}=\zeta_{i}^{\prime} / \rho, \quad i=0,1,
$$

and these local generators of $\mathcal{L}^{\otimes r}$ have all the required properties.

Remark 3.1.2. Set $d:=(u, v)$. Consider the surface

$$
\widetilde{S}_{u, v}=\left\{x s_{0}=\tau^{v / d} s_{1}, y s_{1}=\tau^{u / d} s_{0}\right\} \subset \mathbb{A}_{x, y, \tau}^{3} \times \mathbb{P}_{s_{0}: s_{1}}^{1}
$$


and the family of curves $\widetilde{f}_{u, v}: \widetilde{S}_{u, v} \rightarrow \mathbb{A}_{\tau}^{1}$ induced by the projection. Note that if $d=1$, this is the normalization of $f_{u, v}: S_{u, v} \rightarrow C_{u, v}$.

If the base $T$ is normal, then $\mathcal{X}$ has the following stronger property:

(P) There exist neighbourhoods $T_{0}$ of $t_{0}$ in $T$ and $U$ of $E$ in $\mathcal{X}$, and a regular map $h: T_{0} \rightarrow \mathbb{A}_{\tau}^{1}$, such that $h\left(t_{0}\right)=0$ and $U$ is isomorphic to a neighbourhood of the exceptional curve in the base change of the surface $\widetilde{S}_{u / d, v / d}$ via $h$.

3.2. The model family of curves. Let $C$ be a nodal curve and let $\Delta^{w}$ be a weighted subgraph of $\Gamma_{C}$ satisfying condition $(\mathrm{C} 1)$ of section 2.2. Let $X$ be the curve obtained from $C$ by blowing up the nodes in $\Delta$. Let $\underline{\mathcal{C}} \rightarrow \underline{D}$ be a semiuniversal deformation of the curve $C$, where $\underline{D}$ is the unit polydisc in $\mathbb{C}^{M}$ with coordinates $t_{1}, \ldots, t_{M}$. Observe that this deformation is universal if and only if $C$ is stable. If this is the case, $M$ equals $3 g-3$; otherwise it is strictly larger than $3 g-3$.

We shall construct a finite cover $D_{\Delta^{w}} \rightarrow \underline{D}$ and a family of blow-ups $\pi: \mathcal{X}_{\Delta^{w}} \rightarrow$ $\mathcal{C}_{\Delta^{w}} \rightarrow D_{\Delta^{w}}\left(\right.$ where $\left.\mathcal{C}_{\Delta^{w}}=\underline{\mathcal{C}} \times{ }_{\underline{D}} D_{\Delta^{w}}\right)$ with central fiber $X$ :

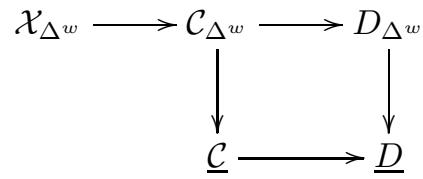

The family $\mathcal{X}_{\Delta^{w}} \rightarrow D_{\Delta^{w}}$ will depend on $C$ and on the weighted graph $\Delta^{w}$; it will be used to construct the universal deformation of any limit $r$-th root $(X, L, \alpha)$ with weighted graph $\Delta^{w}$.

Denote by $E_{1}, \ldots, E_{m}$ the exceptional components of $X$ and by $n_{1}, \ldots, n_{m}$ the corresponding nodes in $C$. For any $i=1, \ldots, m$ let $u_{i}, v_{i}$ be the weights associated to $n_{i}$ and let $\left\{t_{i}=0\right\}$ be the locus in $\underline{D}$ where the node $n_{i}$ persists. We write $\underline{D}=D \times D^{\prime}$, where $D$ is the unit polydisc with coordinates $t_{1}, \ldots, t_{m}$ and $D^{\prime}$ corresponds to the remaining $t_{i}$ 's. Consider

$$
C_{u_{1}, v_{1}} \times \cdots \times C_{u_{m}, v_{m}}=\left\{w_{1}^{u_{1}}=z_{1}^{v_{1}}, \ldots, w_{m}^{u_{m}}=z_{m}^{v_{m}}\right\} \subset \mathbb{A}_{w_{1}, z_{1}, \ldots, w_{m}, z_{m}}^{2 m}
$$

and set

$$
D_{\Delta w}:=\prod_{i=1}^{m} C_{u_{i}, v_{i}} \times D^{\prime}
$$

Consider the morphism $\prod_{i} C_{u_{i}, v_{i}} \rightarrow D$ defined by

$$
t_{i}=w_{i} z_{i} \quad \text { for all } i=1, \ldots, m
$$

and the induced morphism $D_{\Delta^{w}} \rightarrow \underline{D}$. Let $\mathcal{C}_{\Delta^{w}} \rightarrow D_{\Delta^{w}}$ be the pull-back of the family $\underline{\mathcal{C}} \rightarrow \underline{D}$ to $D_{\Delta^{w}}$.

A neighbourhood $V_{i}$ of $n_{i}$ in $\mathcal{C}_{\Delta^{w}}$ can be thought of as

$$
V_{i}=\left\{x y=w_{i} z_{i}, w_{1}^{u_{1}}=z_{1}^{v_{1}}, \ldots, w_{m}^{u_{m}}=z_{m}^{v_{m}}\right\} \subset \mathbb{A}_{x, y, w_{1}, z_{1}, \ldots, w_{m}, z_{m}, t_{m+1}, \ldots, t_{M}}^{M+m+2} .
$$

Now let $\pi: \mathcal{X}_{\Delta^{w}} \rightarrow \mathcal{C}_{\Delta^{w}}$ be the blow-up locally described as follows:

$$
U_{i}=\pi^{-1}\left(V_{i}\right)=\left\{x s_{0}=w_{i} s_{1}, y s_{1}=z_{i} s_{0}, w_{1}^{u_{1}}=z_{1}^{v_{1}}, \ldots, w_{m}^{u_{m}}=z_{m}^{v_{m}}\right\}
$$

in $\mathbb{A}^{M+m+2} \times \mathbb{P}_{s_{0}: s_{1}}^{1}$. Note that $\pi_{\mid U_{i}}: U_{i} \rightarrow V_{i}$ is the blow-up of the ideal $\left(x, w_{i}\right)$, or equivalently of $\left(y, z_{i}\right)$. Now $\mathcal{X}_{\Delta^{w}} \rightarrow D_{\Delta^{w}}$ is a family of blow-ups of $\mathcal{C}_{\Delta^{w}} \rightarrow D_{\Delta^{w}}$, and has $X$ as central fiber. 
Cover each $U_{i}$ by the two affine open subsets $U_{i 0}=\left\{s_{1} \neq 0\right\}$ and $U_{i 1}=\left\{s_{0} \neq 0\right\}$. Set $\eta_{0}:=s_{0} / s_{1}$ and $\eta_{1}:=s_{1} / s_{0}$. Then the total space $\mathcal{X}_{\Delta} w$ has equations

$$
w_{1}^{u_{1}}=z_{1}^{v_{1}}, \ldots, w_{m}^{u_{m}}=z_{m}^{v_{m}}, \quad \begin{cases}x \eta_{0}=w_{i} & \text { in } U_{i 0}, \\ y \eta_{1}=z_{i} & \text { in } U_{i 1},\end{cases}
$$

and $\eta_{0} \eta_{1}=1$ on $U_{i 0} \cap U_{i 1}$. Let $\mathcal{E}$ be the Cartier divisor given by

$$
x^{u_{i}} \text { on } U_{i 0} \text { and } y^{v_{i}} \text { on } U_{i 1} \text {, for } i=1, \ldots, m \text {. }
$$

On $U_{i 0} \cap U_{i 1}$ we have $x^{u_{i}} / y^{v_{i}}=\eta_{1}^{r} \in \mathcal{O}_{\mathcal{X}_{\Delta w}}^{*}\left(U_{i 0} \cap U_{i 1}\right)$. Note that:

$\circ \mathcal{E}$ is effective; its support in each $U_{i}$ is the inverse image via $\pi$ of the locus of the $i$-th node $\left\{x=y=w_{i}=z_{i}\right\}$ in $V_{i} \subset \mathcal{C}_{\Delta w}$;

$\circ \mathcal{E}_{\mid \widetilde{X}}=\sum_{i=1}^{m}\left(u_{i} p_{i}+v_{i} q_{i}\right)$

$\circ \mathcal{O}_{\mathcal{X}_{\Delta} w}(-\mathcal{E})_{\mid E_{i}}$ has degree $r$ for any $i=1, \ldots, m$.

The following result is a global version of Lemma 3.1.1.

Lemma 3.2.1. Let $\mathcal{C} \rightarrow T$ be a family of nodal curves, $\mathcal{N} \in \operatorname{Pic} \mathcal{C}$ and $(\mathcal{X} \rightarrow$ $T, \mathcal{L}, \alpha)$ a limit $r$-th root of $(\mathcal{C}, \mathcal{N})$. Let $t_{0} \in T$, and let $\Delta^{w}$ be the weighted graph of the fiber over $t_{0}$.

Possibly after shrinking $T$, there exist a fiber product diagram

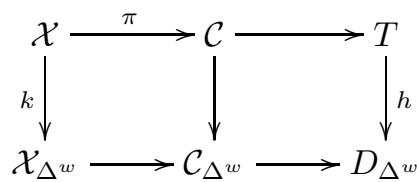

where $h\left(t_{0}\right)=(0, \ldots, 0)$, and an isomorphism $\vartheta: \mathcal{L}^{\otimes r} \rightarrow \pi^{*}(\mathcal{N}) \otimes k^{*}(\mathcal{O}(-\mathcal{E}))$ whose composition with the natural map $\pi^{*}(\mathcal{N}) \otimes k^{*}(\mathcal{O}(-\mathcal{E})) \rightarrow \pi^{*}(\mathcal{N})$ is $\alpha$.

Proof. Call $C$ and $X$ the fibers of $\mathcal{C} \rightarrow T$ and $\mathcal{X} \rightarrow T$ over $t_{0}$. Since $\mathcal{C} \rightarrow T$ is a deformation of $C$, up to shrinking $T$ there is a morphism $\ell: T \rightarrow \underline{D}$ such that $\ell\left(t_{0}\right)=(0, \ldots, 0)$ and $\mathcal{C} \simeq \underline{\mathcal{C}} \times_{D} T$. By Lemma 3.1.1 $\ell$ lifts to $h: T \rightarrow D_{\Delta w}$ and $\mathcal{X} \simeq \mathcal{X}_{\Delta w} \times_{D_{\Delta} w} T$. We define $\vartheta$ to agree with $\alpha$ away from the exceptional components, as $k^{*}(\mathcal{O}(-\mathcal{E}))$ is canonically isomorphic to $\mathcal{O}_{\mathcal{X}}$ in this region. What must be seen is that $\vartheta$ can be extended to all of $\mathcal{X}$. Let $E_{i}$ be an exceptional component of $X$, and let $\zeta_{\mathcal{N}}$ be a generator of $\pi^{*}(\mathcal{N})$ on a neighbourhood of $E_{i}$. Near $E_{i}$, the space $\mathcal{X}$ is obtained from (4) by base change via $h$. Let $U_{0}$ and $U_{1}$ be the inverse images of $U_{i 0}$ and $U_{i 1}$. As in Lemma 3.1.1. we write $x$ and $y$ also to indicate the pull-backs of these functions to $\mathcal{X}$. We know from Lemma 3.1.1 that there are generators $\zeta_{0}$ and $\zeta_{1}$ for $\mathcal{L}^{\otimes r}$, over $U_{0}$ and $U_{1}$, respectively, with the property that

$$
\zeta_{0}=\eta_{1}^{r} \zeta_{1} \text { on } U_{0} \cap U_{1}, \quad \alpha\left(\zeta_{0}\right)=x^{u} \zeta_{\mathcal{N}}, \quad \alpha\left(\zeta_{1}\right)=y^{v} \zeta_{\mathcal{N}},
$$

while $\pi^{*}(\mathcal{N}) \otimes k^{*}(\mathcal{O}(-\mathcal{E}))$ is generated on $U_{0}$ and $U_{1}$ by $\zeta_{0}^{\prime}=\zeta_{\mathcal{N}} \otimes k^{*}\left(x^{u}\right)$ and $\zeta_{1}^{\prime}=\zeta_{\mathcal{N}} \otimes k^{*}\left(y^{v}\right)$. Now we can extend $\vartheta$ across $E_{i}$ by sending $\zeta_{0}$ to $\zeta_{0}^{\prime}$ and $\zeta_{1}$ to $\zeta_{1}^{\prime}$; since $\zeta_{0}^{\prime}=\eta_{1}^{r} \zeta_{1}^{\prime}$, the first of the identities (6) says that this is not ambiguous. On the other hand, since $\zeta_{0}^{\prime}$ maps to $x^{u} \zeta_{\mathcal{N}}$ in $\pi^{*}(\mathcal{N})$, and $\zeta_{1}^{\prime}$ to $y^{v} \zeta_{\mathcal{N}}$, the remaining identities say that the composition of $\vartheta$ with $\pi^{*}(\mathcal{N}) \otimes k^{*}(\mathcal{O}(-\mathcal{E})) \rightarrow \pi^{*}(\mathcal{N})$ agrees with $\alpha$.

Remark 3.2.2. Since $D_{\Delta^{w}}$ equals $C_{u_{1}, v_{1}} \times \cdots \times C_{u_{m}, v_{m}} \times D^{\prime}$, it is normal if and only if, for every $i$, either $u_{i}=1$, or $v_{i}=1$. This is always true if $r=2$ or $r=3$. 
3.3. Construction of universal deformations. We are now in a position to construct the universal deformation of a limit root. We place ourselves in the setup of section 2.4. Let $B$ be a scheme, $f: \mathcal{C} \rightarrow B$ a family of nodal curves and $\mathcal{N} \in$ Pic $\mathcal{C}$ a line bundle of relative degree divisible by $r$.

Fix $b_{0} \in B$, set $C=f^{-1}\left(b_{0}\right)$ and let $\underline{\mathcal{C}} \rightarrow \underline{D}$ be a semi-universal deformation of $C$. There exist a neighbourhood $B_{0}$ of $b_{0}$ in $B$ and a morphism $B_{0} \rightarrow \underline{D}$, $b_{0} \mapsto(0, \ldots, 0)$, such that $\mathcal{C}_{B_{0}} \simeq \underline{\mathcal{C}} \times{ }_{D} B_{0}$.

Let $\xi:=(X, L, \alpha)$ be a limit $r$-th root of $\left(C, N_{b_{0}}\right)$ and let $\Delta^{w}$ be the associated weighted subgraph of $\Gamma_{C}$. Let $\mathcal{X}_{\Delta w} \rightarrow D_{\Delta} w$ be the deformation of $X$ constructed above. Set

$$
U_{\xi}:=B_{0} \times_{\underline{D}} D_{\Delta^{w}},
$$

let $u_{0} \in U_{\xi}$ be the point that maps to $b_{0} \in B_{0}$ and define $\mathcal{C}_{\xi}, \mathcal{X}_{\xi}$ in the natural way. We denote by $\mathcal{N}_{\xi}$ the pull-back of $\mathcal{N}$ under $\mathcal{C}_{\xi} \rightarrow \mathcal{C}_{B_{0}}$; observe that in general $\mathcal{N}_{\xi}$ is not the pull-back of a line bundle on $\mathcal{C}_{\Delta^{w}}$ :

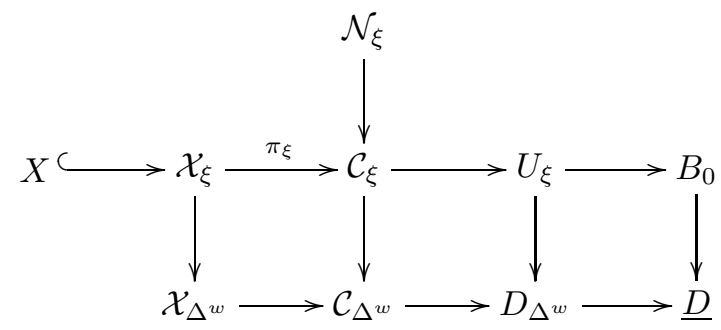

Our goal is to prove that $L$ extends to a line bundle on $\mathcal{X}_{\xi}$, giving a family of limit $r$-th roots of $\mathcal{N}_{\xi}$.

A key step is the next lemma, concerning automorphisms of the above diagram. As usual, when $W \rightarrow Z$ is a morphism of schemes, we write $\operatorname{Aut}_{Z}(W)$ to denote the group of automorphisms of $W$ over $Z$. We shall make use of Lemma 2.3.2 by fixing an orientation on the graph $\Sigma_{X}$ and hence an isomorphism $\operatorname{Aut}_{C}(X) \simeq \mathcal{C}^{1}\left(\Sigma_{X}, \mathbb{C}^{*}\right)$.

Lemma 3.3.1. The action of $G=\operatorname{Aut}_{\underline{D}}\left(D_{\Delta^{w}}\right)$ on $D_{\Delta^{w}}$ lifts to:

a) a natural action on $\mathcal{X}_{\Delta^{w}}$, which leaves the divisor $\mathcal{E}$ invariant;

b) natural actions $G \rightarrow \operatorname{Aut}_{B_{0}}\left(U_{\xi}\right), G \rightarrow \operatorname{Aut}_{B_{0}}\left(\mathcal{C}_{\xi}\right)$, and $G \rightarrow \operatorname{Aut}_{B_{0}}\left(\mathcal{X}_{\xi}\right)$, compatible with the projections $\mathcal{X}_{\xi} \rightarrow \mathcal{C}_{\xi} \rightarrow U_{\xi}$.

Moreover, the homomorphism $G \rightarrow \operatorname{Aut}(X)$ induced by $G \rightarrow \operatorname{Aut}_{B_{0}}\left(\mathcal{X}_{\xi}\right)$ yields an isomorphism $G \stackrel{\sim}{\longrightarrow} \mathcal{C}^{1}\left(\Sigma_{X}, \mu_{r}\right) \subset \operatorname{Aut}_{C}(X)$.

Proof. Automorphisms of $D_{\Delta w}$ over $\underline{D}$ are automorphisms of $C_{u_{1}, v_{1}} \times \cdots \times C_{u_{m}, v_{m}}$ over $D$. Any such automorphism has the form $\left(w_{i}, z_{i}\right) \mapsto\left(\zeta_{i} w_{i}, \zeta_{i}^{-1} z_{i}\right)$ with $\zeta_{i}$ an $r$-th root of unity; thus $G \simeq\left(\mu_{r}\right)^{m}$.

We show that $G$ acts on $\mathcal{X}_{\Delta^{w}}$ and that the action on the central fiber is by inessential automorphisms, giving $G \simeq \mathcal{C}^{1}\left(\Sigma_{X}, \mu_{r}\right) \subset \operatorname{Aut}_{C}(X)$ (cf. Lemma 2.3.2). Fix $\left(\zeta_{1}, \ldots, \zeta_{m}\right) \in\left(\mu_{r}\right)^{m}$. In each $U_{i}$ the space $\mathcal{X}_{\Delta^{w}}$ has equations (44) and the action is given by

$$
\begin{gathered}
\left(x, \eta_{0}, \ldots, w_{i}, \ldots\right) \mapsto\left(x, \zeta_{i} \eta_{0}, \ldots, \zeta_{i} w_{i}, \ldots\right) \text { in } U_{i 0}, \\
\left(y, \eta_{1}, \ldots, z_{i}, \ldots\right) \mapsto\left(y, \zeta_{i}^{-1} \eta_{1}, \ldots, \zeta_{i}^{-1} z_{i}, \ldots\right) \text { in } U_{i 1},
\end{gathered}
$$

hence the induced automorphism of $X$ is inessential and corresponds to $\left(\zeta_{1}, \ldots, \zeta_{m}\right)$ $\in \mathcal{C}^{1}\left(\Sigma_{X}, \mu_{r}\right)$. It is clear that $\mathcal{E}$ is invariant under this action. 
Now, using the universal property of fiber products, it is immediate to see that the action of $G$ lifts to $U_{\xi}, \mathcal{C}_{\xi}$, and $\mathcal{X}_{\xi}$ with the desired properties.

In what follows, if $\sigma \in G$, we shall denote by $\sigma_{U_{\xi}}, \sigma_{\mathcal{C}_{\xi}}$, and $\sigma_{\mathcal{X}_{\xi}}$ the corresponding automorphisms of $U_{\xi}, \mathcal{C}_{\xi}$, and $\mathcal{X}_{\xi}$.

We now address the problem of extending $L$. Recall that there exists an effective Cartier divisor $\mathcal{E}$ on $\mathcal{X}_{\Delta}{ }^{w}$ such that $\mathcal{E}_{\mid \widetilde{X}}=\sum_{i=1}^{m}\left(u_{i} p_{i}+v_{i} q_{i}\right)$ and $\mathcal{O}_{\mathcal{X}_{\Delta} w}(-\mathcal{E})_{\mid E_{i}}$ has degree $r$ for any $i=1, \ldots, m$. Denote by $\mathcal{G}_{\xi}$ the pull-back of $\mathcal{O}_{\mathcal{X}_{\Delta} w}(-\mathcal{E})$ to $\mathcal{X}_{\xi}$. Then part (i) of Lemma 2.3.3 implies that there are an inessential automorphism $\sigma$ of $X$ and an isomorphism $\vartheta: L^{\otimes r} \rightarrow \sigma^{*}\left(\pi_{\xi}^{*}\left(\mathcal{N}_{\xi}\right) \otimes \mathcal{G}_{\xi} \otimes \mathcal{O}_{X}\right)$ such that $\alpha$ is the composition of $\vartheta$ with the pull-back of $\pi_{\xi}^{*}\left(\mathcal{N}_{\xi}\right) \otimes \mathcal{G}_{\xi} \otimes \mathcal{O}_{X} \rightarrow \pi_{\xi}^{*}\left(\mathcal{N}_{\xi}\right) \otimes \mathcal{O}_{X}$. Hence, up to modifying via $\sigma$ the identification of $X$ with the central fiber of $\mathcal{X}_{\xi}$, we can assume that $\alpha: L^{\otimes r} \rightarrow \pi_{b_{0}}^{*}\left(N_{b_{0}}\right)$ agrees with the restriction to $X$ of the natural $\operatorname{map} \pi_{\xi}^{*}\left(\mathcal{N}_{\xi}\right) \otimes \mathcal{G}_{\xi} \rightarrow \pi_{\xi}^{*}\left(\mathcal{N}_{\xi}\right)$.

Shrinking $U_{\xi}$ and $B_{0}$, if necessary, we can extend $L$ to $\mathcal{L}_{\xi} \in \operatorname{Pic} \mathcal{X}_{\xi}$ so that

$$
\mathcal{L}_{\xi}^{\otimes r} \simeq \pi_{\xi}^{*}\left(\mathcal{N}_{\xi}\right) \otimes \mathcal{G}_{\xi},
$$

by Remark 3.0.6. Let $\alpha_{\xi}$ be the composition of this isomorphism with $\pi_{\xi}^{*}\left(\mathcal{N}_{\xi}\right) \otimes \mathcal{G}_{\xi} \rightarrow$ $\pi_{\xi}^{*}\left(\mathcal{N}_{\xi}\right)$. Now $\left(\mathcal{X}_{\xi} \rightarrow U_{\xi}, \mathcal{L}_{\xi}, \alpha_{\xi}\right)$ is a limit $r$-th root of $\mathcal{N}_{\xi}$. Moreover, there is an isomorphism $\widehat{\psi}$ of limit roots between $\xi$ and the fiber of the family $\left(\mathcal{X}_{\xi} \rightarrow U_{\xi}, \mathcal{L}_{\xi}, \alpha_{\xi}\right)$ over $u_{0} \in U_{\xi}$. The pair $\left(\left(\mathcal{X}_{\xi} \rightarrow U_{\xi}, \mathcal{L}_{\xi}, \alpha_{\xi}\right), \widehat{\psi}\right)$ is a universal deformation for $\xi$.

Proposition 3.3.2. Let $p: T \rightarrow B$ be a morphism of schemes, and $(\mathcal{X} \rightarrow T, \mathcal{L}, \beta) a$ limit $r$-th root of $\mathcal{N}_{T}$. Let $t_{0} \in T$ be such that $p\left(t_{0}\right)=b_{0}$, and assume that there is an isomorphism $\widehat{\phi}$ of limit roots between $\xi=(X, L, \alpha)$ and the fiber of $(\mathcal{X} \rightarrow T, \mathcal{L}, \beta)$ over $t_{0}$. Then, possibly after shrinking $T$, the deformation $((\mathcal{X} \rightarrow T, \mathcal{L}, \beta), \widehat{\phi})$ is isomorphic to the pull-back of $\left(\left(\mathcal{X}_{\xi} \rightarrow U_{\xi}, \mathcal{L}_{\xi}, \alpha_{\xi}\right), \widehat{\psi}\right)$ via a unique morphism of $B$-schemes $\gamma: T \rightarrow U_{\xi}$ such that $\gamma\left(t_{0}\right)=u_{0}$; moreover, the isomorphism is unique.

Proof of existence. We know by Lemma 3.2.1 that, up to shrinking $T$, there exists $h: T \rightarrow D_{\Delta} w$ such that $\mathcal{C}_{T} \simeq \mathcal{C}_{\Delta w} \times_{D_{\Delta} w} T$ and $\mathcal{X} \simeq \mathcal{X}_{\Delta^{w}} \times_{D_{\Delta} w} T$. Recall that $U_{\xi}=B_{0} \times_{\underline{D}} D_{\Delta^{w}}$, and set $\gamma=p \times h$. Then $\gamma: T \rightarrow U_{\xi}$ has the property that $\gamma\left(t_{0}\right)=u_{0}$ and that there is a commutative diagram with fiber product squares

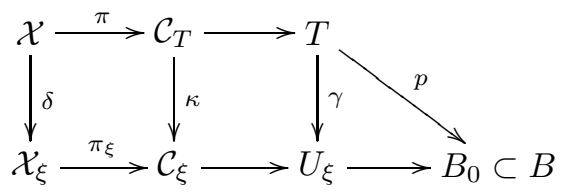

Let $\mathcal{L}^{\prime}$ and $\beta^{\prime}$ be the line bundle and the homomorphism obtained by pulling back $\mathcal{L}_{\xi}$ and $\alpha_{\xi}$ via $\delta$. Note that $\left(\mathcal{X}, \mathcal{L}^{\prime}, \beta^{\prime}\right)$ is a limit $r$-th root of $\mathcal{N}_{T}$, since $\kappa^{*}\left(\mathcal{N}_{\xi}\right)=\mathcal{N}_{T}$ by the commutativity of (8). Moreover, it follows from Lemma 3.2.1 and from the definition of $\mathcal{L}_{\xi}$ that there is an isomorphism $\vartheta: \mathcal{L}^{\otimes r} \rightarrow \mathcal{L}^{\prime \otimes r}$ such that $\beta^{\prime} \circ \vartheta=\beta$.

Now let $\phi: X \rightarrow X_{t_{0}}$ and $\psi: X \rightarrow\left(X_{\xi}\right)_{u_{0}}$ be the isomorphisms of schemes underlying $\widehat{\phi}$ and $\widehat{\psi}$, and set $L^{\prime}=\phi^{*}\left(\mathcal{L}^{\prime}\right)$. Then $\sigma:=\psi^{-1} \circ \delta \circ \phi$ is an inessential automorphism of $X$ with the property that $\sigma^{*}(L)=L^{\prime}$, and $\vartheta$ yields an isomorphism $\chi: L^{\otimes r} \rightarrow \sigma^{*}(L)^{\otimes r}$ such that $\alpha=\sigma^{*}(\alpha) \circ \chi$. Then part ii) of Lemma 2.3.3 says that $\sigma$ belongs to $\mathcal{C}^{1}\left(\Sigma_{X}, \mu_{r}\right)$ which, by Lemma 3.3.1. can be identified with $G=$ $\operatorname{Aut}_{\underline{D}}\left(D_{\Delta w}\right)$. Moreover, Lemma 3.3.1, suitably interpreted, implies that $\psi \circ \sigma=$ 
$\sigma_{\mathcal{X}_{\xi}} \circ \psi$. Thus, replacing $\gamma$ with $\sigma_{U_{\xi}}^{-1} \circ \gamma, \kappa$ with $\sigma_{\mathcal{C}_{\xi}}^{-1} \circ \kappa$, and $\delta$ with $\sigma_{\mathcal{X}_{\xi}}^{-1} \circ \delta$, we may suppose that $\psi=\delta \circ \phi$ (recall that, always by Lemma 3.3.1 $\mathcal{E}$ is $G$-invariant). A consequence is that $\mathcal{L}$ and $\mathcal{L}^{\prime}$ agree at $t_{0}$. On the other hand, $\mathcal{L}^{\otimes r}$ and $\mathcal{L}^{\prime \otimes r}$ are isomorphic via $\vartheta$, so Remark 3.0.6 implies that there is an isomorphism between $\mathcal{L}$ and $\mathcal{L}^{\prime}$ whose $r$-th tensor power is $\vartheta$. At this point we are almost done: $(\mathcal{X}, \mathcal{L}, \beta)$ is certainly the pull-back of $\left(\mathcal{X}_{\xi}, \mathcal{L}_{\xi}, \alpha_{\xi}\right)$. Moreover, $\widehat{\phi}$ is the pull-back of $\widehat{\psi}$, except for one little detail, namely that the corresponding isomorphisms between $L$ and the pull-back of $\mathcal{L}$ might not be equal. However, since the $r$-th tensor powers of these isomorphisms are equal, the ratio between the two is an $r$-th root of unity. To fix this it suffices to multiply the isomorphism between $\mathcal{L}$ and $\mathcal{L}^{\prime}$ by a suitable root of unity.

Proof of uniqueness. Assume that the morphism $p: T \rightarrow B_{0}$ has another lifting $\gamma^{\prime}: T \rightarrow U_{\xi}$ such that $((\mathcal{X}, \mathcal{L}, \beta), \widehat{\phi})$ is isomorphic to the pull-back of $\left(\left(\mathcal{X}_{\xi}, \mathcal{L}_{\xi}, \alpha_{\xi}\right), \widehat{\psi}\right)$ via $\gamma^{\prime}$, and let

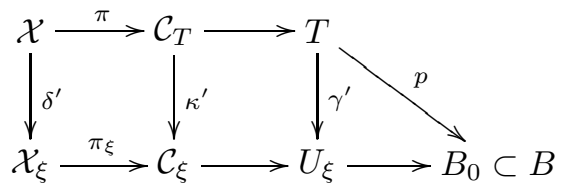

be the corresponding commutative diagram. Call $h$ and $h^{\prime}$ the morphisms $T \rightarrow D_{\Delta} w$ induced, respectively, by $\gamma$ and $\gamma^{\prime}$. Then $h$ and $h^{\prime}$ are both liftings of $T \rightarrow \underline{D}$, so there exists $\sigma \in G$ such that $h^{\prime}=\sigma \circ h$. As $p$ is fixed, this yields that $\gamma^{\prime}=\sigma_{U_{\xi}} \circ \gamma$, and hence also that $\kappa^{\prime}=\sigma_{\mathcal{C}_{\xi}} \circ \kappa$. If we compose the vertical arrows of (9) with the actions of the inverse of $\sigma$ on the respective target spaces, we get another commutative diagram whose vertical arrows are $\delta^{\prime \prime}:=\sigma_{\mathcal{X}_{\xi}}^{-1} \circ \delta^{\prime}, \kappa$, and $\gamma$. Clearly, $\delta^{\prime \prime}$ and $\delta$ differ at most by an automorphism of $\mathcal{X}$ over $\mathcal{C}_{T}$; in other words, there is $\iota \in \operatorname{Aut}_{\mathcal{C}_{T}}(\mathcal{X})$ such that $\delta^{\prime \prime}=\delta \circ \iota$. Moreover, there is an isomorphism between $\mathcal{L}^{\otimes r}$ and $\iota^{*}\left(\mathcal{L}^{\otimes r}\right)$ which is compatible with $\beta$. We need an auxiliary lemma.

Lemma 3.3.3. Let $\iota \in \operatorname{Aut}_{\mathcal{C}_{T}}(\mathcal{X})$. Suppose that there exists an isomorphism $\vartheta: \mathcal{L}^{\otimes r} \rightarrow \iota^{*}\left(\mathcal{L}^{\otimes r}\right)$ such that $\beta=\iota^{*}(\beta) \circ \vartheta$. Then there exists $\tau \in G$ such that, possibly after shrinking $T, \delta \circ \iota=\tau_{\mathcal{X}_{\xi}} \circ \delta$.

Proof of the lemma. This is essentially a version "with parameters" of part (ii) of Lemma 2.3.3. Let $E$ be an exceptional component of $X_{t_{0}}$. Recall from Lemma 3.1.1 that there are open sets $U_{0}$ and $U_{1}$ covering $E$ such that $\mathcal{X}$ is of the form $x \eta_{0}=h_{0}$ in $U_{0}$ and of the form $x \eta_{1}=h_{1}$ in $U_{1}$, where $\eta_{0} \eta_{1}=1$ and $h_{0}, h_{1}$ are functions on a neighbourhood of $t_{0}$ such that $h_{0}^{u}=h_{1}^{v}$. Since $\iota$ is an automorphism over $\mathcal{C}_{T}, \iota^{*}(x)=x$ and $\iota^{*}(y)=y$. Write

$$
\iota^{*}\left(\eta_{0}\right)=\sum_{i \geq 0} a_{i} x^{i}+\sum_{i<0} a_{i} \eta_{0}^{-i}, \quad \iota^{*}\left(\eta_{1}\right)=\sum_{i \geq 0} b_{i} y^{i}+\sum_{i<0} b_{i} \eta_{1}^{-i} .
$$

From $\iota^{*}(x) \iota^{*}\left(\eta_{0}\right)=h_{0}$ we get in particular that $a_{i}=0$ for $i \geq 0$. Similarly, $b_{i}=0$ for $i \geq 0$. On the other hand, $a_{-1}$ and $b_{-1}$ are both different from zero, since $\iota$ restricts to an automorphism of $E$. Starting from $\iota^{*}\left(\eta_{0}\right) \iota^{*}\left(\eta_{1}\right)=1$, another simple power series computation then yields that $a_{i}=b_{i}=0$ for $i<-1$, and that $a_{-1} b_{-1}=1$. Thus $\iota^{*}\left(\eta_{0}\right)=k^{-1} \eta_{0}$ and $\iota^{*}\left(\eta_{1}\right)=k \eta_{1}$, where $k=b_{-1}$ is a unit on a neighbourhood of $t_{0}$. To prove the lemma it suffices to show that $k$ is an $r$-th root of 
unity. Now, Lemma 3.1.1 says that there are generators $\zeta_{i}$ for $\mathcal{L}^{\otimes r}$ on $U_{i}, i=0,1$, and $\zeta_{\mathcal{N}}$ for $\pi^{*}\left(\mathcal{N}_{T}\right)$ on $U_{0} \bigcup U_{1}$, with the property that $\zeta_{0}=\eta_{1}^{r} \zeta_{1}, \beta\left(\zeta_{0}\right)=x^{u} \zeta_{\mathcal{N}}$, and $\beta\left(\zeta_{1}\right)=y^{v} \zeta_{\mathcal{N}}$. Write $\vartheta\left(\zeta_{i}\right)=f_{i} \iota^{*}\left(\zeta_{i}\right), i=0,1$, where

$$
f_{0}=\sum_{i \geq 0} c_{i} x^{i}+\sum_{i<0} c_{i} \eta_{0}^{-i}, \quad f_{1}=\sum_{i \geq 0} d_{i} y^{i}+\sum_{i<0} d_{i} \eta_{1}^{-i} .
$$

From $\beta\left(\zeta_{0}\right)=x^{u} \zeta_{\mathcal{N}}$ and $\beta=\iota^{*}(\beta) \circ \vartheta$ we then get that $x^{u}=x^{u} f_{0}$; this yields that $c_{i}=0$ for $i>0$ and that $c_{0}=1$. Similarly, $d_{i}=0$ for $i>0$, and $d_{0}=1$. On the other hand,

$$
\eta_{1}^{r} f_{1} \iota^{*}\left(\zeta_{1}\right)=\eta_{1}^{r} \vartheta\left(\zeta_{1}\right)=\vartheta\left(\zeta_{0}\right)=f_{0} \iota^{*}\left(\zeta_{0}\right)=f_{0} \iota^{*}\left(\eta_{1}^{r} \zeta_{1}\right)=k^{r} \eta_{1}^{r} f_{0} \iota^{*}\left(\zeta_{1}\right) .
$$

This gives that $d_{0} \eta_{1}^{r}+d_{-1} \eta_{1}^{r-1}+\cdots=k^{r}\left(c_{0} \eta_{1}^{r}+c_{-1} \eta_{1}^{r+1}+\cdots\right)$, and hence in particular that $k^{r}=1$, as desired.

We return to the proof of uniqueness in Proposition 3.3.2. Lemma 3.3.3 implies that $\delta^{\prime}=\sigma_{\mathcal{X}_{\xi}} \circ \tau_{\mathcal{X}_{\xi}} \circ \delta$. On the other hand, $\tau_{\mathcal{C}_{\xi}} \circ \kappa=\kappa$ and $\tau_{U_{\xi}} \circ \gamma=\gamma$, since $\tau_{\mathcal{X}_{\xi}}$ corresponds, via $\delta$, to an automorphism of $\mathcal{X}$ over $\mathcal{C}_{T}$. Thus, replacing the original $\sigma$ with $\sigma \tau$, we may in fact suppose that not only $\gamma^{\prime}=\sigma_{U_{\xi}} \circ \gamma$ and $\kappa^{\prime}=\sigma_{\mathcal{C}_{\xi}} \circ \kappa$, but also $\delta^{\prime}=\sigma_{\mathcal{X}_{\xi}} \circ \delta$. In particular, $\sigma_{\mathcal{X}_{\xi}} \circ \delta \circ \phi=\psi$. However, by the proof of existence, $\psi$ is also equal to $\delta \circ \phi$, so $\sigma_{\mathcal{X}_{\xi}}$ acts trivially on the fiber of $\mathcal{X}_{\xi} \rightarrow U_{\xi}$ at $u_{0}$. Since this action corresponds via $\psi$ to the action of $\sigma$ on $X$, it follows that $\sigma=\mathrm{Id}, \gamma^{\prime}=\gamma$, and $\delta^{\prime}=\delta$. That there is a unique isomorphism $\mathcal{L} \simeq \delta^{*}\left(\mathcal{L}_{\xi}\right)$ which is compatible with $\beta$ and $\delta^{*}\left(\alpha_{\xi}\right)$ and agrees with the given one on the fibers above $t_{0}$ follows from the uniqueness part of Remark 3.0.6. This finishes the proof of Proposition 3.3 .2

Remark 3.3.4. It follows from the construction of $\left(\mathcal{X}_{\xi} \rightarrow U_{\xi}, \mathcal{L}_{\xi}, \alpha_{\xi}\right)$ that this family is a universal deformation for any one of its fibers.

3.4. The moduli scheme of limit roots. We are ready to prove Theorem 2.4.1. Consider the set

$$
\bar{S}_{f}^{r}(\mathcal{N})=\coprod_{b \in B}\left\{\text { limit } r \text {-th roots of }\left(C_{b}, N_{b}\right)\right\} / \sim,
$$

where $\sim$ is the equivalence relation given by isomorphism of limit roots.

Fix $b_{0} \in B$ and a limit $r$-th root $\xi=(X, L, \alpha)$ of $\left(C_{b_{0}}, N_{b_{0}}\right)$. Let $\left(\mathcal{X}_{\xi} \rightarrow\right.$ $\left.U_{\xi}, \mathcal{L}_{\xi}, \alpha_{\xi}\right)$ be the universal deformation of $\xi$ and $B_{0} \subset B$ the open subset of $B$ dominated by $U_{\xi}$.

Recall that, by Lemmas 2.3 .2 and 3.3 .1 there are natural identifications and maps:

$$
\operatorname{Aut}(\xi)=\mathcal{C}^{0}\left(\Sigma_{X}, \mu_{r}\right) \rightarrow \mathcal{C}^{1}\left(\Sigma_{X}, \mu_{r}\right)=G .
$$

Via this homomorphism, the group $\operatorname{Aut}(\xi)$ acts on $U_{\xi}, \mathcal{C}_{\xi}$, and $\mathcal{X}_{\xi}$; if $\sigma \in \operatorname{Aut}(\xi)$, we will denote by $\sigma_{U_{\xi}}, \sigma_{\mathcal{C}_{\xi}}$, and $\sigma_{\mathcal{X}_{\xi}}$ the corresponding automorphisms of these spaces. The universality of the family $\left(\mathcal{X}_{\xi}, \mathcal{L}_{\xi}, \alpha_{\xi}\right)$ shown in Proposition 3.3 .2 implies that, if $\sigma \in \operatorname{Aut}(\xi)$, then $\sigma_{\mathcal{X}_{\xi}}^{*}\left(\mathcal{L}_{\xi}\right) \simeq \mathcal{L}_{\xi}$ and $\sigma_{\mathcal{X}_{\xi}}^{*}\left(\alpha_{\xi}\right) \simeq \alpha_{\xi}$.

Lemma 3.4.1. Let $b \in B_{0}$, let $u_{1}, u_{2} \in U_{\xi}$ be points lying over $b$, and let $\left(X_{1}, L_{1}, \alpha_{1}\right)$, $\left(X_{2}, L_{2}, \alpha_{2}\right)$ be the corresponding limit $r$-th roots of $\left(C_{b}, N_{b}\right)$. The following are equivalent:

(i) there exists $\sigma \in \operatorname{Aut}(\xi)$ such that $\sigma_{U_{\xi}}\left(u_{1}\right)=u_{2}$;

(ii) there exists an isomorphism of limit roots between $\left(X_{1}, L_{1}, \alpha_{1}\right)$ and $\left(X_{2}, L_{2}, \alpha_{2}\right)$. 
Proof. The implication (i) $\Rightarrow$ (ii) is an immediate consequence of what we observed above: if there exists $\sigma \in \operatorname{Aut}(\xi)$ such that $\sigma_{U_{\xi}}\left(u_{1}\right)=u_{2}$, then $\left(\sigma_{\mathcal{X}_{\xi}}\right)_{\mid X_{1}}: X_{1} \rightarrow X_{2}$ is an inessential isomorphism which induces an isomorphism of limit roots.

Conversely, assume that there is an inessential isomorphism $\phi: X_{1} \rightarrow X_{2}$ that induces an isomorphism between the corresponding limit roots. We are going to show first of all that $\phi=\left(\sigma_{\mathcal{X}_{\xi}}\right)_{\mid X_{1}}$ for some $\sigma \in G$, and then that $\sigma$ belongs to the image of $\operatorname{Aut}(\xi)$ in $G$. Let $d \in \underline{D}$ be the image of $b$, and $d_{1}, d_{2} \in D_{\Delta^{w}}$ the images of $u_{1}, u_{2}$. Recall that $G$ is the group of automorphisms of $D_{\Delta w}$ over $\underline{D}$, and that $\underline{D}=D_{\Delta^{w}} / G$. Choose $\tau \in G$ such that $\tau\left(d_{1}\right)=d_{2}$. Then $\tau_{U_{\xi}}\left(u_{1}\right)=u_{2}$ and

$$
\rho:=\phi^{-1} \circ\left(\tau_{\mathcal{X}_{\xi}}\right)_{\mid X_{1}}: X_{1} \longrightarrow X_{1}
$$

is an inessential automorphism of $X_{1}$. Note that $\rho \in \mathcal{C}^{1}\left(\Sigma_{X_{1}}, \mu_{r}\right) \subset \operatorname{Aut}_{C_{b}}\left(X_{1}\right)$ by construction.

Set $\xi_{1}:=\left(X_{1}, L_{1}, \alpha_{1}\right)$. The universal deformation $\left(\mathcal{X}_{\xi_{1}} \rightarrow U_{\xi_{1}}, \mathcal{L}_{\xi_{1}}, \alpha_{\xi_{1}}\right)$ of $\xi_{1}$ is naturally an open subfamily of $\left(\mathcal{X}_{\xi} \rightarrow U_{\xi}, \mathcal{L}_{\xi}, \alpha_{\xi}\right)$ (see Remark 3.3.4). Applying Lemma 3.3.1 with respect to $\xi_{1}$, we see that there is a subgroup $G_{1}$ of $G$ which is naturally identified with $\mathcal{C}^{1}\left(\Sigma_{X_{1}}, \mu_{r}\right) \subset \operatorname{Aut}_{C_{b}}\left(X_{1}\right)$. If $\left\{j_{1}, \ldots, j_{k}\right\} \subset\{1, \ldots, m\}$ are the indices for which the exceptional component $E_{i}$ of $X$ does not deform to $X_{1}$ (namely, in $C_{b}$ the $i$-th node is smoothed), then under the isomorphism $G \simeq\left(\mu_{r}\right)^{m}$, $G_{1}$ is the subgroup of $\left(\zeta_{1}, \ldots, \zeta_{m}\right)$ with $\zeta_{j_{1}}=\cdots=\zeta_{j_{k}}=1$.

Let $\tau^{\prime} \in G_{1}$ be such that $\left(\tau_{\mathcal{X}_{\xi}}^{\prime}\right)_{\mid X_{1}}=\rho$, and set $\sigma:=\tau \circ\left(\tau^{\prime}\right)^{-1}$. Then $\left(\sigma_{\mathcal{X}_{\xi}}\right)_{\mid X_{1}}=\phi$. Note that $\sigma_{\mathcal{X}}^{*}\left(\mathcal{G}_{\xi}\right) \simeq \mathcal{G}_{\xi}$ and $\sigma_{\mathcal{X}_{\xi}}^{*}\left(\pi_{\xi}^{*}\left(\mathcal{N}_{\xi}\right)\right) \simeq \pi_{\xi}^{*}\left(\mathcal{N}_{\xi}\right)$ (this is true for all elements of $G)$. In fact, the first property is easily deduced from (5) and (7); for the second property, it is enough to observe that $\sigma_{\mathcal{C}_{\xi}}^{*}\left(\mathcal{N}_{\xi}\right) \simeq \mathcal{N}_{\xi}$ and that $\pi_{\xi} \circ \sigma_{\mathcal{X}_{\xi}}=\sigma_{\mathcal{C}_{\xi}} \circ \pi_{\xi}$, so $\sigma_{\mathcal{X}_{\xi}}^{*}\left(\pi_{\xi}^{*}\left(\mathcal{N}_{\xi}\right)\right) \simeq \pi_{\xi}^{*}\left(\sigma_{\mathcal{C}_{\xi}}^{*}\left(\mathcal{N}_{\xi}\right)\right) \simeq \pi_{\xi}^{*}\left(\mathcal{N}_{\xi}\right)$.

Now, since $\mathcal{L}_{\xi}^{\otimes r} \simeq \pi_{\xi}^{*}\left(\mathcal{N}_{\xi}\right) \otimes \mathcal{G}_{\xi}$, we get $\sigma_{\mathcal{X}}^{*}\left(\mathcal{L}_{\xi}^{\otimes r}\right) \simeq \mathcal{L}_{\xi}^{\otimes r}$. By construction

$$
\sigma_{\mathcal{X}_{\xi}}^{*}\left(\mathcal{L}_{\xi}\right)_{\mid X_{1}}=\phi^{*}\left(L_{2}\right) \simeq L_{1}=\mathcal{L}_{\xi \mid X_{1}}
$$

so we get $\sigma_{\mathcal{X}_{\xi}}^{*}\left(\mathcal{L}_{\xi}\right) \simeq \mathcal{L}_{\xi}$ by Remark 3.0.6, and $\sigma$ comes from $\operatorname{Aut}(\xi)$.

Proof of Theorem 2.4.1, For every $r$-th root $\xi$ as above we have a well-defined, injective map between B-sets:

$$
\beta_{\xi}: U_{\xi} / \operatorname{Aut}(\xi) \longrightarrow \bar{S}_{f}^{r}(\mathcal{N})
$$

and the image of $\beta_{\xi}$ inherits a complex structure from $U_{\xi} / \operatorname{Aut}(\xi)$. Since $\bar{S}_{f}^{r}(\mathcal{N})$ is covered by subsets of the form $\operatorname{Im} \beta_{\xi}$, in order for these "charts" to define a complex structure on $\bar{S}_{f}^{r}(\mathcal{N})$, the following must hold: if $\xi_{1}$ and $\xi_{2}$ are limit roots such that $\operatorname{Im} \beta_{\xi_{1}}$ and $\operatorname{Im} \beta_{\xi_{2}}$ intersect, then $\beta_{\xi_{2}}^{-1}\left(\operatorname{Im} \beta_{\xi_{1}}\right)$ is open and the composition $\beta_{\xi_{2}}^{-1} \circ \beta_{\xi_{1}}$ is holomorphic. That this is true follows easily from Proposition 3.3.2 Remark 3.3.4, and Lemma 3.4.1 In fact, choose a limit root $\eta$ corresponding to a point in the intersection of $\operatorname{Im} \beta_{\xi_{1}}$ and $\operatorname{Im} \beta_{\xi_{2}}$. Then there are natural (and canonically determined) open immersions $J_{i}: U_{\eta} \hookrightarrow U_{\xi_{i}}$, compatible with the actions of Aut $(\eta)$ and $\operatorname{Aut}\left(\xi_{i}\right)$. Thus $J_{i}$ induces a morphism $\bar{J}_{i}: U_{\eta} / \operatorname{Aut}(\eta) \hookrightarrow U_{\xi_{i}} / \operatorname{Aut}\left(\xi_{i}\right)$, which is an embedding by Lemma 3.4.1. Finally, we have $\beta_{\eta}=\beta_{\xi_{i}} \circ \bar{J}_{i}$.

The analytic morphism $p: \bar{S}_{f}^{r}(\mathcal{N}) \rightarrow B$ has finite fibers. Proposition2.1.2 implies that $p$ is proper, hence it is a finite projective morphism. As $B$ is quasiprojective, so is $\bar{S}_{f}^{r}(\mathcal{N})$. 
Finally, the scheme $\bar{S}_{f}^{r}(\mathcal{N})$ is a coarse moduli space for the functor $\overline{\mathcal{S}}_{f}^{r}(\mathcal{N})$. In fact, for any $B$-scheme $T$, the map $\overline{\mathcal{S}}_{f}^{r}(\mathcal{N})(T) \rightarrow \mathcal{H o m}_{B}\left(T, \bar{S}_{f}^{r}(\mathcal{N})\right)$ is naturally defined as follows. To any limit $r$-th $\operatorname{root}(\mathcal{X} \rightarrow T, \mathcal{L}, \alpha)$ of $\mathcal{N}_{T}$, we associate the morphism $T \rightarrow \bar{S}_{f}^{r}(\mathcal{N})$ which is locally given by Proposition 3.3.2. Clearly, this morphism depends only on the isomorphism class of $(\mathcal{X} \rightarrow T, \mathcal{L}, \alpha)$.

Proof of Proposition 2.4.2. The morphism $p$ is smooth at a point $\xi$ if and only if $U_{\xi} / \operatorname{Aut}(\xi) \simeq B_{0}$, that is, if and only if $\operatorname{Aut}(\xi)=G$. This is equivalent to $b_{1}\left(\Sigma_{X}\right)=0$.

\section{Geometry of the moduli space of limit roots}

4.1. Limit roots for a fixed curve: examples. Let $C$ be a nodal curve and $N \in$ Pic $C$. We denote by $\bar{S}_{C}^{r}(N)$ the zero-dimensional scheme $\bar{S}_{f_{C}}^{r}(N)$, where $f_{C}: C \rightarrow\{p t\}$ is the trivial family. Note that, by Remark 2.4.3. for any family $f: \mathcal{C} \rightarrow B$ whose fiber over $b_{0} \in B$ is $C$ and for any $\mathcal{N} \in \operatorname{Pic} \mathcal{C}$ such that $\mathcal{N}_{\mid C}=$ $N$, the scheme-theoretical fiber over $b_{0}$ of the finite morphism $\bar{S}_{f}^{r}(\mathcal{N}) \rightarrow B$ is isomorphic to $\bar{S}_{C}^{r}(N)$.

We have seen in Remark 2.2.1 that there are $r^{b_{1}\left(\Gamma_{C}\right)}$ weighted subgraphs of $\Gamma_{C}$ satisfying conditions $(\mathrm{C} 1)$ and $(\mathrm{C} 2)$. Let $\Delta^{w}$ be one of them. Recall that $\Delta^{w}$ carries the weights $u_{i}, v_{i}$ on each edge, and that its supporting graph $\bar{\Delta}$ is a subgraph of $\Gamma_{C}$. As usual, denote by $g^{\nu}$ the genus of the normalization of $C$. The set of nodes $\Delta$ determines a partial normalization $\widetilde{\pi}: \widetilde{X} \rightarrow C$ of $C$, and the weights determine a line bundle $\widetilde{\pi}^{*}(N)\left(-\sum_{i}\left(u_{i} p_{i}+v_{i} q_{i}\right)\right)$ on $\tilde{X}$. The dual graph of $\widetilde{X}$ coincides with $\Gamma_{C} \backslash \Delta$, so we have $r^{2 g^{\nu}+b_{1}\left(\Gamma_{C} \backslash \Delta\right)}$ choices for a line bundle $\widetilde{L} \in \operatorname{Pic} \widetilde{X}$ such that $\widetilde{L}^{\otimes r} \simeq \widetilde{\pi}^{*}(N)\left(-\sum_{i}\left(u_{i} p_{i}+v_{i} q_{i}\right)\right)\left(r^{2 g^{\nu}}\right.$ choices for the pull-back of $\widetilde{L}$ to the normalization of $\widetilde{X}$, and $r^{b_{1}\left(\Gamma_{C} \backslash \Delta\right)}$ choices for the gluings at the nodes). Each choice of $\widetilde{L}$ gives a point $(X, L, \alpha)$ in $\bar{S}_{C}^{r}(N)$ with weighted graph $\Delta^{w}$.

Lemma 4.1.1. The geometric multiplicity of the connected component of $\bar{S}_{C}^{r}(N)$ supported on $(X, L, \alpha)$ is $r^{b_{1}\left(\Sigma_{X}\right)}=r^{b_{1}\left(\Gamma_{C}\right)-b_{1}\left(\Gamma_{C} \backslash \Delta\right)}$.

Proof. Recall that $\Sigma_{X}$ is the graph whose vertices are the connected components of $\widetilde{X}$ and whose edges are the exceptional components of $X$. The connected component of $\bar{S}_{C}^{r}(N)$ corresponding to $(X, L, \alpha)$ is isomorphic to the fiber over the origin of the morphism $D_{\Delta^{w}} / \operatorname{Aut}(X, L, \alpha) \rightarrow \underline{D}$ (see section 3). If $X$ has $m$ exceptional components and $\gamma$ is the number of connected components of $\widetilde{X}$, then the order of ramification of $D_{\Delta^{w}} / \operatorname{Aut}(X, L, \alpha) \rightarrow \underline{D}$ over the origin is $r^{m} / r^{\gamma-1}=r^{b_{1}\left(\Sigma_{X}\right)}$. Now note that $\Sigma_{X}$ is obtained from $\Gamma_{C}$ by contracting all edges in $\Gamma_{C} \backslash \Delta$, thus $b_{1}\left(\Sigma_{X}\right)=b_{1}\left(\Gamma_{C}\right)-b_{1}\left(\Gamma_{C} \backslash \Delta\right)$.

In conclusion, using Remark 2.2.1, we have that the length of $\bar{S}_{C}^{r}(N)$ is

$$
\sum_{\Delta^{w} \in \partial^{-1}\left([\underline{d}]_{r}\right)} r^{2 g^{\nu}+b_{1}\left(\Gamma_{C} \backslash \Delta\right)} \cdot r^{b_{1}\left(\Gamma_{C}\right)-b_{1}\left(\Gamma_{C} \backslash \Delta\right)}=r^{b_{1}\left(\Gamma_{C}\right)} \cdot r^{2 g^{\nu}+b_{1}\left(\Gamma_{C}\right)}=r^{2 g},
$$

as expected. Here, as usual, $[\underline{d}]_{r}$ stands for the modulo $r$ multidegree of $N$.

Remark 4.1.2. Consider a limit root $(C, L)$ having $C$ itself as an underlying curve; then the scheme $\bar{S}_{C}^{r}(N)$ is reduced at the point $(C, L)$. 
Remark 4.1.3. By construction $D_{\Delta^{w}} \rightarrow \underline{D}$ depends only on $C$ and on $\Delta^{w}$, while $\operatorname{Aut}(X, L, \alpha)$ depends only on $\Sigma_{X}$ and $r$, by Lemma 2.3.2. Thus

- the scheme structure on $\bar{S}_{C}^{r}(N)$ depends only on $[\underline{d}]_{r}$.

Example 4.1.4 (Compact type). Let $C=\bigcup_{j} C_{j}$ be a curve of compact type. Then $b_{1}\left(\Gamma_{C}\right)=0$, hence there is a unique weighted subgraph of $\Gamma_{C}$ satisfying $(\mathrm{C} 1)$ and $(\mathrm{C} 2)$ (which depends on $[\underline{d}]_{r}$ by the previous remark). So there is a unique blow-up $X$ of $C$ ( $X$ is of compact type) with a divisor $D=\sum_{i}\left(u_{i} p_{i}+v_{i} q_{i}\right)$, such that every limit root in $\bar{S}_{C}^{r}(N)$ is of type $(X, L, \alpha)$ with $L_{\mid \widetilde{X}}^{\otimes r} \simeq \tilde{\pi}^{*}(N)(-D)$. In particular, $X=C$ if and only if $[\underline{d}]_{r}=0$. Note also that $\bar{S}_{C}^{r}(N)$ is reduced.

Example 4.1.5. Let $C$ be a curve of genus $g$ with two smooth components $C_{1}, C_{2}$ and three nodes $n_{1}, n_{2}, n_{3}$ :

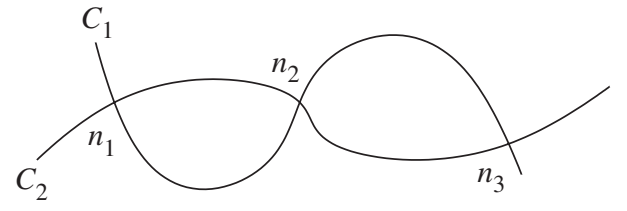

$C$

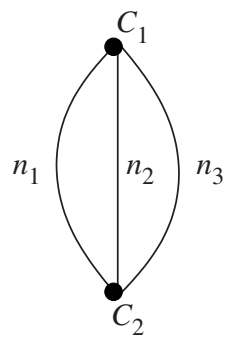

$\Gamma_{C}$

We describe $\bar{S}_{C}^{3}\left(\mathcal{O}_{C}\right)$. Since $b_{1}\left(\Gamma_{C}\right)=2, \Gamma_{C}$ must have $3^{2}=9$ weighted subgraphs satisfying $(\mathrm{C} 1)$ and $(\mathrm{C} 2)$. One is $\emptyset$. This choice corresponds to points of type $(C, L)$ with $L^{\otimes 3} \simeq \mathcal{O}_{C}$; there are $9 \cdot 3^{2 g^{\nu}}$ of them (with $g^{\nu}=g-2$ ), all reduced points in $\bar{S}_{C}^{3}\left(\mathcal{O}_{C}\right)$.

For each $\Delta$ consisting of two edges $\left\{n_{j}, n_{k}\right\}$ (where $j, k \in\{1,2,3\}$ ), we have two possible choices for the weights:

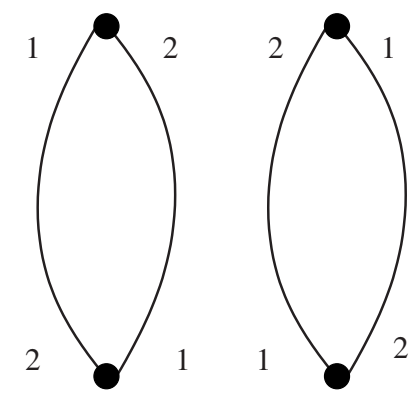


Denote by $X_{j, k}$ the curve obtained by blowing up $\Delta$.

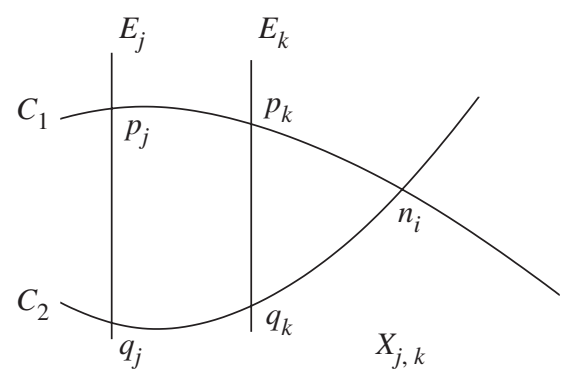

We get points of type $\left(X_{j, k}, L\right)$ where $L_{\mid \widetilde{X}_{j, k}}^{\otimes 3} \simeq \mathcal{O}_{\widetilde{X}_{j, k}}\left(-2 p_{j}-q_{j}-p_{k}-2 q_{k}\right)$ or $L_{\mid \widetilde{X}_{j, k}}^{\otimes 3} \simeq \mathcal{O}_{\widetilde{X}_{j, k}}\left(-p_{j}-2 q_{j}-2 p_{k}-q_{k}\right)$, depending on the weights. Since $\Gamma_{C} \backslash \Delta$ is contractible, we have $b_{1}\left(\Gamma_{C} \backslash \Delta\right)=0$, so these points have multiplicity 9 in $\bar{S}_{C}^{3}\left(\mathcal{O}_{C}\right)$; there are $6 \cdot 3^{2 g^{\nu}}$ of them.

Finally, when $\Delta=\left\{n_{1}, n_{2}, n_{3}\right\}$, we have two possible choices for the weights:
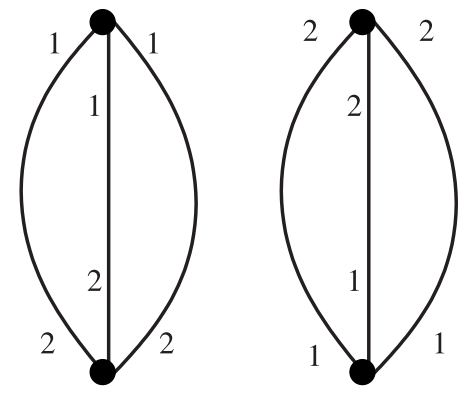

Denote by $X_{1,2,3}$ the corresponding curve.

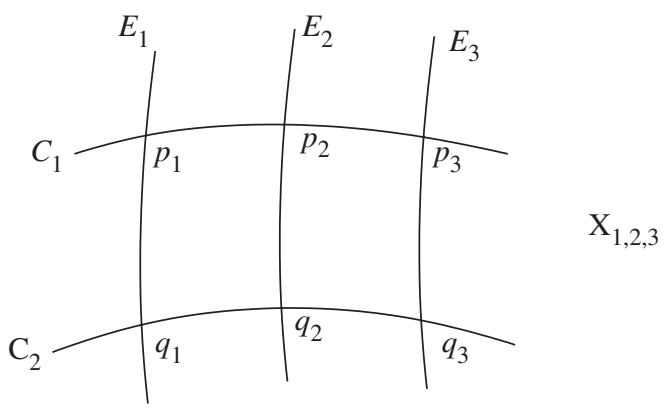

We get points $\left(X_{1,2,3}, L\right)$, where $L_{\mid \widetilde{X}_{1,2,3}}^{\otimes 3} \simeq \mathcal{O}_{\widetilde{X}_{1,2,3}}\left(-p_{1}-p_{2}-p_{3}-2 q_{1}-2 q_{2}-2 q_{3}\right)$ or $L_{\mid \widetilde{X}_{1,2,3}}^{\otimes 3} \simeq \mathcal{O}_{\widetilde{X}_{1,2,3}}\left(-2 p_{1}-2 p_{2}-2 p_{3}-q_{1}-q_{2}-q_{3}\right)$. There are $2 \cdot 3^{2 g^{\nu}}$ of these points, each of them with multiplicity 9 in $\bar{S}_{C}^{3}\left(\mathcal{O}_{C}\right)$.

4.2. Higher spin curves. Let $\bar{M}_{g, n}$ be the moduli space, and $\overline{\mathcal{M}}_{g, n}$ the moduli stack, of stable $n$-pointed curves of genus $g$. Let $\omega_{u_{n}}$ be the relative dualizing sheaf, and $\Sigma_{1}, \ldots, \Sigma_{n}$ the tautological sections, of the universal curve $u_{n}: \overline{\mathcal{C}}_{g, n} \rightarrow \overline{\mathcal{M}}_{g, n}$. 
Fix integers $l, m_{1}, \ldots, m_{n}$ such that $r \mid l(2 g-2)+m_{1}+\cdots+m_{n}$, and set $\underline{m}:=$ $\left\{m_{1}, \ldots, m_{n}\right\}$ and

$$
\omega_{\underline{m}}^{l}:=\omega_{u_{n}}^{\otimes l}\left(m_{1} \Sigma_{1}+\cdots+m_{n} \Sigma_{n}\right) \in \operatorname{Pic} \overline{\mathcal{C}}_{g, n} .
$$

We introduce a contravariant functor

$$
\overline{\mathcal{S}}_{g, n}^{r, l, \underline{m}}:\{\text { schemes }\} \longrightarrow\{\text { sets }\},
$$

encoding the moduli problem for limit $r$-th roots of $\omega_{\underline{m}}^{l}$ over $\overline{\mathcal{M}}_{g, n}$, as follows. Look at pairs $(f: \mathcal{C} \rightarrow B, \xi)$ consisting of a family $f: \mathcal{C} \rightarrow \bar{B}$ of $n$-pointed genus $g$ curves and a limit root $\xi$ of $\left(\mathcal{C}, \omega_{f}^{\otimes l}\left(\sum m_{i} \sigma_{i}\right)\right)$, where $\sigma_{1}, \ldots, \sigma_{n}$ are the tautological sections of $f$ and $\omega_{f}$ is the relative dualizing sheaf. Two such pairs $(f: \mathcal{C} \rightarrow B, \xi)$ and $\left(f^{\prime}: \mathcal{C}^{\prime} \rightarrow B, \xi^{\prime}\right)$ are considered equivalent if there is an isomorphism $\kappa: \mathcal{C} \rightarrow \mathcal{C}^{\prime}$ of families of $n$-pointed curves and an isomorphism, as limit roots of $\left(\mathcal{C}, \omega_{f}^{\otimes l}\left(\sum m_{i} \sigma_{i}\right)\right)$, between $\xi$ and $\kappa^{*}\left(\xi^{\prime}\right)$ (observe that, if $\sigma_{1}^{\prime}, \ldots, \sigma_{n}^{\prime}$ are the tautological sections of $f^{\prime}$, then $\omega_{f}^{\otimes l}\left(\sum m_{i} \sigma_{i}\right)$ and $\kappa^{*}\left(\omega_{f^{\prime}}^{\otimes l}\left(\sum m_{i} \sigma_{i}^{\prime}\right)\right)$ are canonically isomorphic). Note that this is clearly a coarser equivalence relation than the one given by isomorphism of limit roots over a fixed family of curves.

Then, for every scheme $B$, we define $\overline{\mathcal{S}}_{g, n}^{r, l, \underline{m}}(B)$ to be the set of all equivalence classes of pairs $(f: \mathcal{C} \rightarrow B, \xi)$ as above. Arguing exactly as in the proof of Theorem 2.4.1 we obtain:

Theorem 4.2.1. The functor $\overline{\mathcal{S}}_{g, n}^{r, l, \underline{m}}$ is coarsely represented by a projective scheme $\bar{S}_{g, n}^{r, l, \underline{m}}$, finite over $\bar{M}_{g, n}$.

We call $\bar{\varphi}: \bar{S}_{g, n}^{r, l, \underline{m}} \rightarrow \bar{M}_{g, n}$ the structure morphism. For any stable $n$-pointed curve $\left(C, \sigma_{1}, \ldots, \sigma_{n}\right)$, the fiber of $\bar{\varphi}$ over $[C]$ is

$$
\bar{S}_{C}^{r}\left(\omega_{C}^{\otimes l}\left(m_{1} \sigma_{1}+\cdots+m_{n} \sigma_{n}\right)\right) / \operatorname{Aut}\left(C, \sigma_{1}, \ldots, \sigma_{n}\right) .
$$

When we consider stable curves without markings (i.e., when $n=0$ ), we denote by $\bar{S}_{g}^{r, l}$ the corresponding moduli space, and by $\bar{S}_{C}^{r, l}$ the fiber of $\bar{\varphi}$ over $[C] \in \bar{M}_{g}$. The scheme $\bar{S}_{g}^{2,1}$ is the moduli space of spin curves, constructed in 8$]$.

Denote by $S_{g, n}^{r, l, \underline{m}}$ the open subscheme of $\bar{S}_{g, n}^{r, l, \underline{m}}$ parametrizing points $(C, L)$ with $C$ stable, and let $\varphi: S_{g, n}^{r, l, \underline{m}} \rightarrow \bar{M}_{g, n}$ be the natural morphism.

Pairs $(C, L)$ with $C$ a smooth curve and $L \in$ Pic $C$ such that $L^{\otimes r} \simeq \omega_{C}$ are generally called $r$-spin curves. Their moduli space admits a natural stratification by loci parametrising line bundles $L$ with increasing $h^{0}(C, L)$, so that the largest locus corresponds to "effective $r$-spin curves"; we refer to the recent paper of A. Polishchuk 21] for more details and open problems related to this interesting aspect.

Several modular compactifications of the moduli space of $r$-spin curves, and more generally of $S_{g, n}^{r, l, \underline{m}}$, have been introduced by T. J. Jarvis [14, 15, 16], by means of rank 1 , torsion-free sheaves.

The next statement summarizes well-known results relating line bundles and torsion-free sheaves of rank 1 .

Proposition 4.2.2. Let $B$ be an integral scheme and $f: \mathcal{C} \rightarrow B$ a family of nodal curves. 
(I) Let $\pi: \mathcal{X} \rightarrow \mathcal{C}$ be a family of blow-ups of $\mathcal{C}$ and let $\mathcal{L} \in$ Pic $\mathcal{X}$ be a line bundle having degree 1 on every exceptional component. Then $\pi_{*}(\mathcal{L})$ is a relatively torsion-free sheaf of rank 1 , flat over $B$.

(II) Conversely, suppose that $\mathcal{F}$ is a relatively torsion-free sheaf of rank 1 on $\mathcal{C}$, flat over $B$. Then there exist a family $\pi: \mathcal{X} \rightarrow \mathcal{C}$ of blow-ups of $\mathcal{C}$ and a line bundle $\mathcal{L} \in \operatorname{Pic} \mathcal{X}$ having degree 1 on all exceptional components, such that $\mathcal{F} \simeq \pi_{*}(\mathcal{L})$.

(III) Let $\pi: \mathcal{X} \rightarrow \mathcal{C}, \pi^{\prime}: \mathcal{X}^{\prime} \rightarrow \mathcal{C}$ be families of blow-ups of $\mathcal{C}$ and $\mathcal{L} \in$ Pic $\mathcal{X}$, $\mathcal{L}^{\prime} \in \operatorname{Pic} \mathcal{X}^{\prime}$ line bundles having degree 1 on every exceptional component. Then $\pi_{*}(\mathcal{L}) \simeq \pi_{*}^{\prime}\left(\mathcal{L}^{\prime}\right)$ if and only if there exists an isomorphism $\sigma: \mathcal{X} \stackrel{\sim}{\rightarrow} \mathcal{X}^{\prime}$ over $\mathcal{C}$ such that $\mathcal{L} \simeq \sigma^{*}\left(\mathcal{L}^{\prime}\right)$.

Proof. (I) is a local property, see [14, Proposition 3.1.2.

(II) is a consequence of G. Faltings' local characterization of torsion-free sheaves [11; see [14, $\S 3.1$ and 3.2, in particular Theorem 3.2.2.

(III) The "if" part is clear. To prove the converse, it suffices to note that there is an isomorphism $\tau: \mathcal{X} \rightarrow \operatorname{Proj}\left(\bigoplus_{k \geq 0} \operatorname{Sym}^{k}\left(\pi_{*}(\mathcal{L})\right)\right)$ of spaces over $\mathcal{C}$, with the property that $\tau^{*}(\mathcal{O}(1))$ is isomorphic to $\mathcal{L}$, and similarly for $\mathcal{X}^{\prime}$ and $\mathcal{L}^{\prime}$.

Jarvis introduces the notion of an $r$-th root sheaf of a line bundle $N \in \operatorname{Pic} C$, which is a rank 1 , torsion-free sheaf $\mathcal{F}$ on $C$ together with a homomorphism $\beta: \mathcal{F}^{\otimes r} \rightarrow N$ with the following properties (see [16], Definition 2.3):

○ $r \operatorname{deg} \mathcal{F}=\operatorname{deg} N$

- $\beta$ is an isomorphism on the locus where $\mathcal{F}$ is locally free;

- at any node of $C$ where $\mathcal{F}$ is not locally free, the length of the cokernel of $\beta$ is $r-1$.

Such an $r$-th root sheaf is always of the form $\pi_{*}(L)$, where $X$ is the curve obtained from $C$ by blowing-up the nodes where $\mathcal{F}$ is not locally free, $\pi: X \rightarrow C$ is the natural morphism and $(X, L, \bar{\beta})$ is a limit $r$-th root of $(C, N)$. It is easy to see, using Proposition 4.2.2, that the moduli functor of $r$-th root sheaves of $\omega_{\underline{m}}^{l}$ over $\bar{M}_{g, n}$ is isomorphic to $\overline{\mathcal{S}}_{g, n}^{r, l, \underline{m}}$ (see [14], 3.1 and 4.2.2, and [16], 2.2.2 and page 38). Hence we have the following:

Theorem 4.2.3. The scheme $\bar{S}_{g, n}^{r, l, \underline{m}}$ is isomorphic over $\bar{M}_{g, n}$ to the coarse moduli space of the stack $\operatorname{RoOT}_{g, n}^{1 / r}\left(\omega_{\underline{m}}^{l}\right)$ (in the notation of [15]) of $r$-th root sheaves of $\omega_{\underline{m}}^{l}$.

Observe that, exactly as in [15, one could construct different (less singular) compactifications of $S_{g, n}^{r, l, \underline{m}}$ (and more generally of $S_{f}^{r}(\mathcal{N})$ ) in two ways:

(a) requiring, in the definition of a family of limit roots, that property $(\mathrm{P})$ of Remark 3.1.2 holds; this amounts to considering only "not too singular" families of limit roots;

(b) attaching to a limit $r$-th root the data of a set of limit $d$-th roots for any positive $d$ dividing $r$, i.e., considering "coherent nets of roots". Set-theoretically, this amounts to attaching to a limit $r$-th $\operatorname{root} \xi=(X, L, \alpha)$ the data of the gluings for $\xi^{m}$ for any integer $m$ dividing $r$. 


\section{Higher spin Curves in the universal PiCARd SCheme}

5.1. Compactifying the Picard functor. Assume $g \geq 3$ and let $P_{d, g} \rightarrow M_{g}^{0}$ be the degree $d$ universal Picard variety 5 whose fiber over a smooth curve $C$ is $\operatorname{Pic}^{d} C$, the variety of line bundles of degree $d$ on $C$. Let $\bar{P}_{d, g} \rightarrow \bar{M}_{g}$ be the modular compactification constructed in [5; $\bar{P}_{d, g}$ is an integral, normal projective scheme. Its fiber over $C \in \bar{M}_{g}^{0}$ is denoted by $\bar{P}_{C}^{d}$ and gives a compactification of (a finite number of copies of) the generalized Jacobian of $C$. The moduli properties of $\bar{P}_{d, g}$ are described in [5], section 8. Its boundary points are of the same nature as limit roots: they correspond to line bundles on quasistable curve: 6 having degree 1 on exceptional components.

The moduli space of higher spin curves over $M_{g}^{0}$ naturally embeds in $P_{d, g}$ (see Lemma-Definition 5.2.1); its projective closure in $\bar{P}_{d, g}$ is thus a new compactification which we shall now study.

We recall from [5] a few basic properties of $\bar{P}_{d, g}$, which, as $\bar{M}_{g}$, is constructed by means of Geometric Invariant Theory (see [13; in this section we freely use the language of [19]). We begin with some useful conventions and numerical preliminaries. Let $d$ be an integer; we denote by $\underline{d}$ an element of $\mathbb{Z}^{\gamma}$ whose entries add up to $d$, that is, $\underline{d}=\left\{d_{1}, \ldots, d_{\gamma}\right\}$ and $\sum d_{i}=d$. We say that $\underline{d}$ is divisible by some integer $r$ if all of its entries are; if that is the case, we write $\underline{d} \equiv 0(\bmod r)$. If $q$ is a rational number, we set $q \underline{d}=\left\{q d_{1}, \ldots, q d_{\gamma}\right\}$, and we say that $q \underline{d}$ is integer if $q d_{i}$ is integer for every $i$.

For any nodal curve $X$, we denote by $X_{1}, \ldots, X_{\gamma}$ its irreducible components and set $k_{i}=\# X_{i} \cap \overline{X \backslash X_{i}}$. Let $M$ be a line bundle of degree $d$ on $X$; denote by $\underline{d}=\operatorname{deg} M=\left\{d_{1}, \ldots, d_{\gamma}\right\}$ its multidegree, where $d_{i}:=\operatorname{deg}_{X_{i}} M$. For the dualizing sheaf of $X$ we use the special notation $w_{i}:=\operatorname{deg}_{X_{i}} \omega_{X}=2 g\left(X_{i}\right)-2+k_{i}$ and

$$
\underline{w}_{X}:=\underline{\operatorname{deg}} \omega_{X}=\left\{w_{1}, \ldots, w_{\gamma}\right\} .
$$

Similarly, for a subcurve $Z$ of $X$, we set $k_{Z}:=\# Z \cap \overline{X \backslash Z}, w_{Z}:=\operatorname{deg}_{Z} \omega_{X}=$ $2 g(Z)-2+k_{Z}$ and $d_{Z}:=\operatorname{deg}_{Z} M$.

Let $X$ be a quasistable curve. As usual, we denote by $E_{i}$ its exceptional components and set $\widetilde{X}=\overline{X \backslash \bigcup_{i} E_{i}}$ (thus $\widetilde{X}$ is a partial normalization of $C$ ).

Definition 5.1.1. Let $X$ be a quasistable curve and $M \in \operatorname{Pic} X$. We shall say that the multidegree $\underline{d}$ of $M$ is balanced if $\operatorname{deg}_{E} M=1$ on every exceptional component $E$ of $X$ and if, for every subcurve $Z$ of $X$, the following inequality (known as Basic Inequality) holds:

$$
\left|d_{Z}-d \frac{w_{Z}}{2 g-2}\right| \leq \frac{k_{Z}}{2}
$$

We shall say that $\underline{d}$ is stably balanced if it is balanced and if for every subcurve $Z$ of $X$ such that

$$
d_{Z}-d \frac{w_{Z}}{2 g-2}=-\frac{k_{Z}}{2},
$$

we have that $Z$ contains $\widetilde{X}$.

Similarly, we shall say that the line bundle $M$ is balanced (or stably balanced) if its multidegree is.

\footnotetext{
${ }^{5} M_{g}^{0}$ (and likewise $\bar{M}_{g}^{0}$ ) stands for the locus of curves with a trivial automorphism group.
}

${ }^{6} \mathrm{~A}$ quasistable curve $X$ is a blow-up of a stable curve $C$. We call $C$ the stable model of $X$. 
It is easy to see that if $\underline{d}$ is balanced and $Z$ is a connected component of $\widetilde{X}$, then $d_{Z}-d \frac{w_{Z}}{2 g-2}=-\frac{k_{Z}}{2}$. Hence if $\underline{d}$ is stably balanced, $\widetilde{X}$ is connected.

We shall need the following elementary

Lemma 5.1.2. Let $X$ be a quasistable curve.

(i) Let $M \in \operatorname{Pic} X$ be such that $M^{\otimes r} \simeq \omega_{X}^{\otimes l}$ for some integers $r$ and $l$; then $M$ is stably balanced.

(ii) $M \in \operatorname{Pic} X$ is balanced (stably balanced) if and only if $M \otimes \omega_{X}^{\otimes t}$ is balanced (stably balanced) for some integer $t$.

(iii) Assume that the stable model of $X$ is irreducible and let $M \in \operatorname{Pic} X$ be a line bundle having degree 1 on all exceptional components. Then $M$ is stably balanced.

The goal of the following definitions is to measure the nonseparatedness of the Picard functor.

Definition 5.1.3. Let $X$ be a nodal curve and $T \in \operatorname{Pic} X$; we say that $T$ is a twister if there exists a one-parameter smoothing $\mathcal{X} \rightarrow S$ of $X$ such that

$$
T \simeq \mathcal{O}_{\mathcal{X}}\left(\sum_{i=1}^{\gamma} a_{i} X_{i}\right) \otimes \mathcal{O}_{X}
$$

for some Cartier divisor $\sum_{i=1}^{\gamma} a_{i} X_{i}$ on $\mathcal{X}$ (where $a_{i} \in \mathbb{Z}$ ). The set of all twisters of $X$ is denoted by $\operatorname{Tw}(X)$.

Definition 5.1.4. Consider two balanced line bundles $M \in \operatorname{Pic} X$ and $M^{\prime} \in$ Pic $X^{\prime}$, where $X$ and $X^{\prime}$ are quasistable curves.

We say that $M$ and $M^{\prime}$ are equivalent if there exists a semistable curve $Y$ dominating 7 both $X$ and $X^{\prime}$ and a twister $T \in \operatorname{Tw}(Y)$ such that, denoting by $M_{Y}$ and $M_{Y}^{\prime}$ the pull-backs of $M$ and $M^{\prime}$ to $Y$, we have

$$
M_{Y}^{\prime} \simeq M_{Y} \otimes T
$$

In particular, for any automorphism $\sigma$ of $X, \sigma^{*}(M)$ is equivalent to $M$.

To say that $M$ and $M^{\prime}$ are equivalent is to say that their pull-backs to $Y$ are both limits of the same family of line bundles. More precisely, let $\mathcal{Y} \longrightarrow S$ be a one-parameter smoothing of $Y$ such that the twister $T$ of Definition [5.1.4 is $T=\mathcal{O}_{\mathcal{Y}}(D) \otimes \mathcal{O}_{Y}$, with $\operatorname{Supp} D \subset Y$. Let $\mathcal{M} \in \operatorname{Pic} \mathcal{Y}$ be such that $\mathcal{M}_{\mid Y} \simeq M_{Y}$; then $\mathcal{M} \otimes \mathcal{O}_{\mathcal{Y}}(D) \otimes \mathcal{O}_{Y} \simeq M_{Y}^{\prime}$ so that $M_{Y}$ and $M_{Y}^{\prime}$ are both limits of the family corresponding to $\mathcal{M}^{*}$ (recall that the "*" denotes restriction away from the central fiber). Therefore, the line bundles $M_{Y}$ and $M_{Y}^{\prime}$ (and likewise $M$ and $M^{\prime}$ ) must be identified in any proper completion of the Picard scheme over $\bar{M}_{g}$.

Remark 5.1.5. Let $M, M^{\prime} \in \operatorname{Pic} X$ be stably balanced. Then a simple numerical checking shows that they are equivalent if and only if there exists an automorphism $\sigma$ of $X$ such that $M^{\prime} \simeq \sigma^{*}(M)$. If the stable model of $X$ has no nontrivial automorphisms, then $M$ and $M^{\prime}$ are equivalent if and only if $M_{\mid \widetilde{X}} \simeq M_{\mid \widetilde{X}}^{\prime}$.

Fix a large $d(d \geq 20(g-1)$ would work $)$, set $s=d-g$ and consider the Hilbert scheme $\mathrm{Hilb}_{\mathbb{P}^{s}}^{d, g}$ of connected curves of degree $d$ and genus $g$ in $\mathbb{P}^{s}$; the group

\footnotetext{
${ }^{7}$ We say that a semistable curve $Y$ dominates a nodal curve $X$ if it can be obtained from $X$ by a finite sequence of blow-ups.
} 
$S L(s+1)$ naturally acts on $\operatorname{Hilb}_{\mathbb{P} s}^{d, g}$. Recall that there exist linearizations for such an action such that the following facts hold.

Theorem 5.1.6 ([5]). Let $X \subset \mathbb{P}^{s}$ be a connected curve of genus $g$.

(1) The Hilbert point of $X$ is GIT-semistable if and only if $X$ is quasistable and $\mathcal{O}_{X}(1)$ is balanced.

(2) The Hilbert point of $X$ is GIT-stable if and only if $X$ is quasistable and $\mathcal{O}_{X}(1)$ is stably balanced.

(3) Assume that the Hilbert points of $X$ and $X^{\prime} \subset \mathbb{P}^{s}$ are GIT-semistable. Then they are GIT-equivalent if and only if $\mathcal{O}_{X}(1)$ and $\mathcal{O}_{X^{\prime}}(1)$ are equivalent.

Proof. We have assembled together, for convenience, various results from [5]: for (1) see Propositions 3.1 and 6.1 (the "only if" part is due to D. Gieseker [13]); for (2) see Lemma 6.1. The "only if" of part (3) is Lemma 5.2; the "if" is a direct consequence of the existence of the GIT quotient as a projective scheme (see below).

Then let $\bar{P}_{d, g}$ be the GIT-quotient of the locus of all GIT-semistable points in $\mathrm{Hilb}_{\mathbb{P} s}^{d, g}$; it is a normal, integral scheme, flat over $\bar{M}_{g}^{0}$ (Theorem 6.1 in [5]).

The above result permits a modular interpretation of $\bar{P}_{d, g}$ as a modular compactification of the universal Picard variety. Let $X$ be a quasistable curve and let $M \in \operatorname{Pic} X$ be very ample of degree $d$. By Theorem 5.1.6, if we embed $X$ in $\mathbb{P}^{s}$ by $M$, the Hilbert point of $X$ is GIT-semistable if and only if $M$ is balanced. Thus $\bar{P}_{d, g}$ parametrizes equivalence classes of balanced line bundles on quasistable curves.

Associating to $M \in \operatorname{Pic} X$ the stable model $C$ of $X$ yields the natural morphism $\bar{P}_{d, g} \rightarrow \bar{M}_{g} ; \bar{P}_{C}^{d}$ denotes its fiber over $[C] \in \bar{M}_{g}$.

Recall (Theorem 6.1 in [5]) that $\bar{P}_{C}^{d}$ is a finite union of $g$-dimensional irreducible components, one for each stably balanced multidegree (of degree $d$ ) on $C$ :

$$
\bar{P}_{C}^{d}=\bigcup_{\underline{d} \frac{\text { stably }}{\text { balanced }}} \bar{P}_{C}^{\underline{d}} .
$$

Moreover, if $C$ has no nontrivial automorphisms, every component of $\bar{P}_{C}^{d}$ contains a copy of the generalized Jacobian of $C$ as a dense open subscheme:

$$
\operatorname{Pic} \stackrel{d}{C} \subset \bar{P} \frac{d}{C}
$$

where $\operatorname{Pic}^{\underline{d}} C$ is the variety of line bundles on $C$ having multidegree $\underline{d}$. If $C$ is irreducible or of compact type, then $\bar{P}_{C}^{d}$ is irreducible.

Remark 5.1.7. By [5], Lemma 8.1, for any integer $t, \bar{P}_{d, g}$ and $\bar{P}_{d+t(2 g-2), g}$ are isomorphic over $\bar{M}_{g}$ via the morphism $M \mapsto M \otimes \omega_{X}^{\otimes t}$ for any $M \in \operatorname{Pic} X$. Hence we can assume that $\bar{P}_{d, g}$ exists for any $d \in \mathbb{Z}$, even if it is constructed only for $d \gg 0$. Moreover, $M$ is balanced, or stably balanced, if and only if $M \otimes \omega_{X}^{\otimes t}$ is (by part (ii) of Lemma 5.1.2).

5.2. A new compactification of higher spin curves. Let $C$ be a stable curve and $L \in \operatorname{Pic} C$ an $r$-th root of $\omega_{C}^{\otimes l}$; then, by part (ii) of Lemma 5.1.2, $L$ is stably 
balanced, therefore, by part (2) of [5.1.6, it is identified with a point of $\bar{P}_{l(2 g-2) / r, g}$. More precisely, consistently with section 4.2, we denote by $S_{g}^{r, l}$ the open subscheme of $\bar{S}_{g}^{r, l}$ corresponding to pairs $(C, L)$ as above. Obviously, $S_{g}^{r, l}$ is the coarse moduli space for the similarly defined functor $\mathcal{S}_{g}^{r, l}$. We have:

Lemma-Definition 5.2.1. There is a natural embedding

$$
\chi: S_{g}^{r, l} \hookrightarrow \bar{P}_{l(2 g-2) / r, g} .
$$

We define $\widehat{S}_{g}^{r, l}$ to be the closure, in the projective variety $\bar{P}_{l(2 g-2) / r, g}$, of the image of $\chi$, and

$$
\widehat{\varphi}: \widehat{S}_{g}^{r, l} \longrightarrow \bar{M}_{g}
$$

to be the natural morphism.

Proof. The set-theoretic inclusion has already been defined before the statement. To complete the proof, note that there is an obvious inclusion of functors

$$
\mathcal{S}_{g}^{r, l} \hookrightarrow \overline{\mathcal{P}}_{l(2 g-2) / r, g},
$$

and hence the desired embedding follows from [5], Proposition 8.1(1).

This defines a new compactification of the moduli space of higher spin curves, which we shall compare with the previously constructed $\bar{S}_{g}^{r, l}$. As for $\bar{S}_{g}^{r, l}$, the boundary points of $\widehat{S}_{g}^{r, l}$ correspond to line bundles on quasistable curves, having degree 1 on exceptional components. Here is a more precise description.

Proposition 5.2.2. The points of $\widehat{S}_{g}^{r, l}$ are in bijective correspondence with equivalence classes of balanced line bundles $M \in \operatorname{Pic} X$ such that $X$ is a quasistable curve of genus $g$ and there exists a twister $T$ on $X$ for which the following relation holds:

$$
M^{\otimes r} \simeq \omega_{X}^{\otimes l} \otimes T .
$$

Proof. Let $\lambda \in \widehat{S}_{g}^{r, l}$. Since $\lambda$ belongs to the closure of (smooth) $r$-spin curves, there exist a representative $M \in \operatorname{Pic} X$ of $\lambda$, a one-parameter smoothing $f: \mathcal{X} \rightarrow S$ of $X$, and a line bundle $\mathcal{M}$ on $\mathcal{X}$, such that $\mathcal{M} \otimes \mathcal{O}_{X} \simeq M$ and such that

$$
\left(\mathcal{M}^{*}\right)^{\otimes r} \simeq\left(\omega_{f}^{*}\right)^{\otimes l}
$$

Then we have

$$
\mathcal{M}^{\otimes r} \simeq \omega_{f}^{\otimes l} \otimes \mathcal{O}_{\mathcal{X}}(D),
$$

where $D$ is a Cartier divisor supported on the central fiber. Finally, the line bundle $T=\mathcal{O}_{\mathcal{X}}(D) \otimes \mathcal{O}_{X}$ is a twister of $X$; therefore, restricting the above relation to $X$, we are done.

Now we prove the converse: let $X$ be a quasistable curve of genus $g$ and let $T \in \operatorname{Tw}(X)$ be such that $\left(\underline{\operatorname{deg}} T+l \underline{w}_{X}\right) r^{-1}$ is integer and balanced. Observe that $\omega_{X}^{\otimes l} \otimes T$ has degree divisible by $r$ on each irreducible component of $X$, so it admits some $r$-th root $M \in \operatorname{Pic} X$. Let us show that $M \in \widehat{S}_{g}^{r, l}$.

$M$ is balanced, so it defines a point in $\bar{P}_{d, g}$. Moreover, by the definition of twister, there exist a 1-parameter smoothing $f: \mathcal{X} \rightarrow S$ of $X$ and a Cartier divisor $D$, supported on the central fiber, such that

$$
\mathcal{O}_{\mathcal{X}}(D) \otimes \mathcal{O}_{X}=T
$$


Then $M$ is an $r$-th root of $\left(\omega_{f}^{\otimes l} \otimes \mathcal{O}_{\mathcal{X}}(D)\right) \otimes \mathcal{O}_{X}$, and by Remark 3.0.6 it has a (unique) extension to $\mathcal{M} \in \operatorname{Pic} \mathcal{X}$ such that

$$
\mathcal{M}^{\otimes r} \simeq \omega_{f}^{\otimes l} \otimes \mathcal{O}_{\mathcal{X}}(D) .
$$

Hence $M$ is a limit of smooth $r$-spin curves, and, as such, it lies in $\widehat{S}_{g}^{r, l}$.

Remark 5.2.3. The proposition highlights the combinatorial nature of $\widehat{S}_{g}^{r, l}$ by saying that the points of $\widehat{S}_{g}^{r, l}$ are determined by all twisters $T$ on $X$ such that $r^{-1} \operatorname{deg}\left(T \otimes \omega_{X}^{\otimes l}\right)$ is an integer and balanced. It is easy to see that this is equivalent to the following properties of $\underline{\operatorname{deg}} T$ :

(i) $\operatorname{deg}_{E_{i}} T=r$ for every exceptional component $E_{i}$ of $X$;

(ii) $\operatorname{deg}_{X_{j}} T \equiv\left(-l w_{j}\right)(\bmod r)$ for every nonexceptional component $X_{j}$ of $X$;

(iii) $\left|\operatorname{deg}_{Z} T\right| \leq \frac{k_{Z} r}{2}$ for every subcurve $Z$ of $X$.

5.3. Fiberwise description. Consider again the embedding $\chi$ defined in LemmaDefinition 5.2.1. As $S_{g}^{r, l}$ is dense in $\bar{S}_{g}^{r, l}$ (and in $\widehat{S}_{g}^{r, l}$ by definition), $\chi$ will be viewed as a birational map

$$
\chi: \bar{S}_{g}^{r, l} \rightarrow \widehat{S}_{g}^{r, l} .
$$

For any stable curve $C$ of genus $g$, we denote by $\widehat{S}_{C}^{r, l}$ the fiber of $\widehat{\varphi}: \widehat{S}_{g}^{r, l} \rightarrow \bar{M}_{g}$ over $[C]$. Throughout this section we assume that the automorphism group of $C$ is trivial. If $C$ is smooth, then $\widehat{S}_{C}^{r, l}=\bar{S}_{C}^{r, l}$ is the reduced, zero-dimensional scheme parametrizing $r$-th roots of $\omega_{C}^{\otimes l}$.

We have a first, easy result relating the two above moduli spaces.

Lemma 5.3.1. Let $(X, L, \alpha) \in \bar{S}_{g}^{r, l}$ be a limit root and let $C$ be a stable curve.

(i) The map $\chi$ is regular at every point $(X, L, \alpha)$ such that $L$ is balanced.

(ii) If every limit root in $\bar{S}_{C}^{r, l}$ is balanced (for instance, if $C$ is irreducible), then the restriction $\chi_{\mid \bar{S}_{C}^{r, l}}: \bar{S}_{C}^{r, l} \rightarrow \widehat{S}_{C}^{r, l}$ is surjective. In particular, $\operatorname{dim} \widehat{S}_{C}^{r, l}=$ 0 .

Proof. The argument for (i) is analogous to the one proving Theorem 5.1.6. The requirement that $L$ be balanced naturally defines a subfunctor of $\overline{\mathcal{S}}_{g}^{r, l}$, which has a natural inclusion in the functor $\overline{\mathcal{P}}_{l(2 g-2) / r, g}$. Hence the statement follows from Proposition 8.1 of [5].

Now (ii). We just proved that $\chi$ is defined on $\bar{S}_{C}^{r, l}$ and that, for any $\xi \in \bar{S}_{C}^{r, l}$ with $\xi=(X, L, \alpha)$, the image $\chi(\xi)$ is the class defined by $L$ in $\widehat{S}_{C}^{r, l}$. Let $\lambda \in \widehat{S}_{C}^{r, l}$ and choose a representative $M \in \operatorname{Pic} X$ for $\lambda$, where $X$ is some blow-up of $C$; pick a one-parameter smoothing of $X, f: \mathcal{X} \rightarrow S$, and $\mathcal{M} \in \operatorname{Pic} \mathcal{X}$ such that

$$
\left(\mathcal{M}^{*}\right)^{\otimes r} \simeq\left(\omega_{f^{*}}\right)^{\otimes l}
$$

("*" means away from the special fiber). Then by Proposition 2.1.2, up to replacing $S$ by a finite (ramified) covering, the family $\left(f^{*}: \mathcal{X}^{*} \rightarrow S^{*}, \mathcal{M}^{*}\right)$ determines a unique limit root $\xi \in \bar{S}_{C}^{r, l}$. By continuity, $\chi(\xi)=\lambda$, hence surjectivity. The fact that (ii) applies to irreducible curves descends from Lemma 5.1.2, (iii). 
Observe that, if $(X, L, \alpha)$ and $(X, L, \beta)$ are two balanced limit roots of $\omega_{C}^{\otimes l}$, then they are identified by $\chi$ to the same point in $\widehat{S}_{g}^{r, l}$, regardless of their being isomorphic or not as limits roots (cf. Lemma 2.3.3 part (ii)).

The main difference between $\widehat{S}_{g}^{r, l}$ and $\bar{S}_{g}^{r, l}$ is that, if $r \geq 3$, the morphism $\widehat{\varphi}$ is not finite. First we have

Lemma 5.3.2. Let $Z$ be an irreducible component of $\widehat{S}_{C}^{r, l}$. Then

$$
\operatorname{dim} Z \leq b_{1}\left(\Gamma_{C}\right) .
$$

In particular, if $C$ is of compact type, then $\operatorname{dim} \widehat{S}_{C}^{r, l}=0$.

Proof. Let $Z$ be an irreducible component of $\widehat{S}_{C}^{r, l}$ and let $\lambda \in Z$. By Proposition 5.2.2, there exists a representative $L \in \operatorname{Pic} X$ for $\lambda$ such that $L^{\otimes r} \simeq \omega_{X}^{\otimes l} \otimes T$ for a twister $T \in \operatorname{Tw}(X)$. For every given $T$ there are obviously finitely many $L \in \operatorname{Pic} X$ satisfying this relation. We claim that the twisters $T$ vary in algebraic varieties of dimension at most $b_{1}\left(\Gamma_{C}\right)$.

Let $\nu: X^{\nu} \rightarrow X$ be the normalization, $T$ a twister, and set $G:=\nu^{*} T \in \operatorname{Pic} X^{\nu}$. Then every $T^{\prime} \in \operatorname{Tw}(X)$ such that $\nu^{*} T^{\prime}=G$ has the same multidegree as $T$, and hence all the $r$-th roots of $\omega_{X}^{\otimes l} \otimes T^{\prime}$ lie in $\widehat{S}_{C}^{r, l}$ (see Remark [5.2.3). Under the map $\nu^{*}: \operatorname{Pic} X \rightarrow \operatorname{Pic} X^{\nu}$ the fiber of $G$ is a $\left(\mathbb{C}^{*}\right)^{b}$, where

$$
b=b_{1}\left(\Gamma_{X}\right)=b_{1}\left(\Gamma_{C}\right)
$$

now, $T^{\prime}$ moves in such a fiber, and hence in a variety of dimension at most $b_{1}\left(\Gamma_{C}\right)$. On the other hand, the varying of $G$ does not contribute to the dimension of $Z$ : it is in fact easy to see that the set $\nu^{*}(\mathrm{Tw} X)$ (where $G$ ranges) is a discrete subset of $\operatorname{Pic} X^{\nu}$. Therefore we are done.

Finally, if $C$ is of compact type, then $b_{1}\left(\Gamma_{C}\right)=0$, so the last statement is a special case of the first.

Proposition 5.3.3. Let $C=C_{1} \cup C_{2}$ with $C_{i}$ smooth and $\# C_{1} \cap C_{2}=k \geq 2$. Then $\operatorname{dim} \widehat{S}_{C}^{r, l}=k-1$, with the following list of exceptions, where $\operatorname{dim} \widehat{S}_{C}^{r, l}=0$ :

(i) $r=2$;

(ii) $k=2, l \underline{w}_{C} \equiv 0(\bmod r)$;

(iii) $k=3, r=4, l \underline{w}_{C} \equiv(2,2)(\bmod 4)$;

(iv) $k=4, r=3, \underline{w}_{C} \equiv 0(\bmod 3)$.

Proof. In the four exceptional cases listed in the statement, it is easy to check that all limit roots in $\bar{S}_{C}^{r, l}$ are balanced, hence by Lemma 5.3.1, part (ii), we have $\operatorname{dim} \widehat{S}_{C}^{r, l}=0$.

Denote by $n_{1}, \ldots, n_{k}$ the nodes of $C$, and by $\nu: C^{\nu} \rightarrow C$ the normalization. We divide the proof into three steps.

Step 1. Fix a twister $T$ on $C$. Let $T^{\prime} \in \operatorname{Pic} C$ be such that $\nu^{*}\left(T^{\prime}\right)=\nu^{*}(T)$; then $T^{\prime}$ is a twister.

Let $\mathcal{C} \rightarrow S$ be such that $T=\mathcal{O}_{\mathcal{C}}\left(\sum_{i} a_{i} C_{i}\right) \otimes \mathcal{O}_{C}$, and suppose that $\mathcal{C}$ has an $A_{h_{j}-1}$ singularity in $n_{j}$. Introduce the universal deformation $\underline{\mathcal{C}} \rightarrow \underline{D}$ of $C$, where $\underline{D}$ is a smooth polydisc. Assume, as usual, that the locus where the nodes $n_{1}, \ldots, n_{k}$ are preserved is defined by the vanishing of the first $k$ coordinates on $\underline{D}$. Consider 
the family $\mathcal{C}^{\prime} \rightarrow S$ obtained by pulling back $\underline{\mathcal{C}}$ via the morphism

$$
\begin{aligned}
S \longrightarrow \underline{D}, \\
t \mapsto\left(c_{1} t^{h_{1}}, \ldots, c_{k} t^{h_{k}}, 0, \ldots, 0\right),
\end{aligned}
$$

where $t$ is the local coordinate on $S$ and the $c_{j} \in \mathbb{C}^{*}$ are arbitrary. Then $\mathcal{C}^{\prime}$, just like $\mathcal{C}$, has an $A_{h_{j}-1}$ singularity at $n_{j}$; therefore $T^{\prime}:=\mathcal{O}_{\mathcal{C}^{\prime}}\left(\sum_{i} a_{i} C_{i}\right) \otimes \mathcal{O}_{C}$ is a line bundle and thus a twister. Finally, it is easy to see that $T^{\prime}$ has gluing datum $c_{j}$ over $n_{j}$. The conclusion is that, for any gluing assignment of $\nu^{*} T$ over the nodes of $C$, we obtain a twister, as desired.

From now on we exclude cases (i),..., (iv) above.

Step 2. There exists a nonzero twister $T_{0}$ on $C$ such that the $r$-th roots of $\omega_{C}^{\otimes l} \otimes T_{0}$ are stably balanced and, in particular, correspond to points of $\widehat{S}_{C}^{r, l}$.

Pick $k$ nonzero integers $b_{1}, \ldots, b_{k}$ having the same sign. As usual, denote by $p_{1}, \ldots, p_{k}$ (respectively, $q_{1}, \ldots, q_{k}$ ) the inverse images in $C_{1}$ (respectively, in $C_{2}$ ) of the nodes. Define $G \in \operatorname{Pic} C^{\nu}$ by

$$
G_{\mid C_{1}}=\mathcal{O}_{C_{1}}\left(\sum_{i=1}^{k} b_{i} p_{i}\right), \quad G_{\mid C_{2}}=\mathcal{O}_{C_{2}}\left(-\sum_{i=1}^{k} b_{i} q_{i}\right) .
$$

Then there exists a twister $T$ on $C$ such that $\nu^{*} T=G$. In fact, set $h:=$ $\operatorname{lcm}\left(\left|b_{1}\right|, \ldots,\left|b_{k}\right|\right)$ and $h_{i}:=h /\left|b_{i}\right|$. Consider a one-parameter smoothing $\mathcal{C} \rightarrow S$ of $C$ having an $A_{h_{i}-1}$ singularity in $n_{i}$ for $i=1, \ldots, k$. Set $D:=h C_{2}$ if the $b_{i}$ are positive, $D:=-h C_{2}$ if the $b_{i}$ are negative. Then $D$ is Cartier and $T:=\mathcal{O}_{\mathcal{C}}(D) \otimes \mathcal{O}_{C}$ is a twister such that $T_{\mid C_{1}}=\mathcal{O}_{C_{1}}\left(b_{1} p_{1}+\cdots+b_{k} p_{k}\right)$ and $T_{\mid C_{2}}=\mathcal{O}_{C_{2}}\left(-b_{1} q_{1}-\cdots-b_{k} q_{k}\right)$, that is, $\nu^{*} T=G$.

Now set $s=\sum b_{i}$; obviously $|s| \geq k$. By Proposition 5.2.2. Step 2 will be proved by solving the following numerical problem.

Find an integer $s$ such that

(1) $|s| \geq k$,

(2) $l w_{1}+s \equiv 0(\bmod r)$ and $l w_{2}-s \equiv 0(\bmod r)$,

(3) $|s|<\frac{k r}{2}$,

where (2) and (3) are parts (ii) and (iii) of Remark 5.2.3, and we ask for strict inequality in (3) because we want the degree to be stably balanced.

Write $s=r j-l w_{1}$ for some integer $j$. It finally suffices to find an integer $j$ so that (1) and (3) above are satisfied, that is, $k \leq\left|r j-l w_{1}\right|<\frac{k r}{2}$. In other words, we are reduced to showing that the set

$$
\left(-\frac{k}{2}+\frac{l w_{1}}{r},-\frac{k}{r}+\frac{l w_{1}}{r}\right] \cup\left[\frac{k}{r}+\frac{l w_{1}}{r}, \frac{k}{2}+\frac{l w_{1}}{r}\right)
$$

contains an integer $j$ (provided that cases (i),.., (iv) are excluded). The proof of this fact is elementary and easy, therefore we omit it.

Step 3. Steps 1 and 2 imply the proposition.

Pick a twister $T_{0}$ as in Step 2. By Proposition 5.2.2 and Remark 5.2.3, the set of all such $T_{0}$ is characterized by some numerical conditions on their multidegree, hence on the multidegree of their pull-back to the normalization of $C$. Therefore, by Step 1 , if there exists one $T_{0}$, then there exists a $b_{1}\left(\Gamma_{C}\right)$-dimensional family $\mathcal{F}$ of them: $\mathcal{F} \simeq\left(\mathbb{C}^{*}\right)^{b_{1}\left(\Gamma_{C}\right)}$ is the entire fiber of $\nu^{*}$ over $\nu^{*}\left(T_{0}\right)$. This yields that for every $T \in \mathcal{F}$ the $r$-th roots of $\omega_{C}^{\otimes l} \otimes T$ lie in $\widehat{S}_{C}^{r, l}$, and correspond to different points, 
because they are stably balanced. We conclude that $\widehat{S}_{C}^{r, l}$ contains subvarieties of dimension $b_{1}\left(\Gamma_{C}\right)=k-1$; by Lemma 5.3 .2 we are done.

5.4. Comparison results. We begin with the case $r=2$, which is essentially already known, as we will explain. Under this condition limit roots are always balanced.

Lemma 5.4.1. Assume that $r=2$. Let $C$ be a stable curve and let $(X, L, \alpha) \in$ $\bar{S}_{g}^{2, l}$ be a limit square root of $\left(C, \omega_{C}^{\otimes l}\right)$. Then $L$ is balanced, and the orbit of the corresponding Hilbert point is closed in the GIT-semistable locus.

Proof. Fix a subcurve $Z$ of $X$, let $k_{Z}$ and $w_{Z}$ be as usual, and let $k_{Z}^{\prime}$ be the number of exceptional components of $X$ intersecting $Z$ and not contained in $Z$. Then we have

$$
k_{Z}^{\prime} \equiv l k_{Z} \quad(\bmod 2),
$$

$k_{Z}^{\prime} \leq k_{Z}$ and $2 d_{Z}=l w_{Z}-k_{Z}^{\prime}$. The Basic Inequality (see Definition 5.1.1) for $Z$ simplifies to $k_{Z}^{\prime} \leq k_{Z}$, which is always satisfied, thus $L$ is balanced and the Hilbert point is GIT-semistable (Theorem [5.1.6). Moreover, if equality holds, every node on $Z \cap \overline{X \backslash Z}$ is exceptional. Then Lemma 6.1 of [5] applies, giving that the Hilbert point has closed orbit, as stated.

In view of Lemma 5.3.1, one obtains that $\chi: \bar{S}_{g}^{2, l} \rightarrow \widehat{S}_{g}^{2, l}$ is regular for all $l$; moreover, $\chi$ is bijective because of the last assertion of the previous lemma (cf. [12]).

When $r \geq 3$ the situation is quite different, for, in general, neither the birational map $\chi: \bar{S}_{g}^{r, l} \longrightarrow \widehat{S}_{g}^{r, l}$ nor its inverse are regular, as we shall presently see.

Theorem 5.4.2. Let $r \geq 3$.

(i) $\chi$ is regular in codimension 1 .

(ii) If $r$ does not divide $2 l$, then $\chi$ is not regular in codimension 2. If $r$ divides $2 l$, then $\chi$ is not regular in codimension 3 , unless $r=4$ and $l \equiv 2(\bmod 4)$.

If $r=4$ and $l \equiv 2(\bmod 4)$, then $\chi$ is not regular in codimension 4 .

(iii) If $r \geq 5$ and $g \geq 7$, or $r=3,4$ and $g \geq 10$, then $\chi^{-1}$ is not regular in codimension 1 .

Proof. First of all, recall that $\bar{\varphi}: \bar{S}_{g}^{r, l} \rightarrow \bar{M}_{g}$ is finite. By Lemma 5.3.1 $\chi$ is regular over the locus of irreducible curves in $\bar{M}_{g}$. Let $U \subset \bar{S}_{g}^{r, l}$ be the inverse image of the locus of curves of compact type in $\bar{M}_{g}$. Then, by Proposition 2.4.2 applied to $B=\bar{M}_{g}, U$ is normal, so $\chi$ is regular in codimension 1 on $U$, hence regular in codimension 1 on $\bar{S}_{g}^{r, l}$. This settles (i).

For (ii) we use Proposition 5.3.3, which describes boundary strata in $\bar{M}_{g}$ over which $\widehat{\varphi}: \widehat{S}_{g}^{r, l} \rightarrow \bar{M}_{g}$ is not finite. Since $\bar{\varphi}$ is finite, $\chi$, being dominant, cannot be regular over such strata.

In case $r$ does not divide $2 l$ we consider the locus of curves $C=C_{1} \cup C_{2}$ with $C_{i}$ smooth, $\# C_{1} \cap C_{2}=2$ and $C_{1}$ of genus 1 (hence $C_{2}$ of genus $g-2$ ). Then $l \underline{w}_{C}=(2 l, 2 l(g-2)) \not \equiv 0(\bmod r)$, so by Proposition $5.3 .3 \hat{\varphi}$ is not finite over this locus. 
If $r$ divides $2 l$ and $(r, l) \neq(4,2+4 j)$, again by Proposition 5.3.3, $\widehat{\varphi}$ is not finite over all codimension 3 loci whose general curve is a union of 2 smooth components meeting at 3 points.

Finally, if $r=4$ and $l \equiv 2(\bmod 4), \widehat{\varphi}$ is not finite over all codimension 4 loci whose general curve is a union of 2 smooth components meeting at 4 points.

(iii) Consider $\xi=(X, L, \alpha) \in \bar{S}_{C}^{r, l}$ and a one-parameter smoothing $(f: \mathcal{X} \rightarrow$ $S, \mathcal{L})$ of $\xi$. Then the moduli morphism $S^{*} \rightarrow \widehat{S}_{g}^{r, l}$ completes to a morphism $S \rightarrow$ $\widehat{S}_{g}^{r, l}$. Denote by $\lambda \in \widehat{S}_{C}^{r, l}$ the image of $s_{0} \in S$. Clearly, if $\chi^{-1}$ is defined at $\lambda$, then $\chi^{-1}(\lambda)=\xi$. We shall exhibit, for a suitable choice of $C, r$ distinct points $\xi_{1}, \ldots, \xi_{r} \in \bar{S}_{C}^{r, l}$ and one-parameter deformations $f_{1}, \ldots, f_{r}$ as above, such that the associated $\lambda_{i}$ all lie in the same irreducible component of $\widehat{S}_{C}^{r, l}$. This implies that $\chi^{-1}$ is not defined at $\lambda_{i}$, as the $\xi_{i}$ are irreducible components of $\bar{S}_{C}^{r, l}$.

Let $C=C_{1} \cup C_{2}$ with $C_{i}$ smooth, $C_{1} \simeq \mathbb{P}^{1}$ and $\# C_{1} \cap C_{2}=k$ (hence $C$ has genus $g \geq k-1$ and $w_{1}=k-2$ ).

We treat the case $r \geq 5, g \geq 7$ and $l=1$, the remaining cases being similar. Let $k=7$, fix 5 nodes $n_{1}, \ldots, n_{5}$ of $C$ and consider the weighted subgraph of $\Gamma_{C}$ given by these five nodes with weights $u_{1}=\cdots=u_{5}=1$ and $v_{1}=\cdots=v_{5}=r-1$. This weighted graph satisfies conditions $(\mathrm{C} 1)$ and $(\mathrm{C} 2)$ of section 2.2 with respect to $\omega_{C}$.

Let $X$ be the blow-up of $C$ at $n_{1}, \ldots, n_{5}$. Fix $L_{i} \in \operatorname{Pic} C_{i}$ such that

$$
L_{1}^{\otimes r} \simeq\left(\omega_{X}\right)_{\mid C_{1}} \otimes \mathcal{O}_{C_{1}}\left(-\sum_{j=1}^{5} p_{j}\right) \quad \text { and } \quad L_{2}^{\otimes r} \simeq\left(\omega_{X}\right)_{\mid C_{2}} \otimes \mathcal{O}_{C_{2}}\left(-\sum_{j=1}^{5}(r-1) q_{j}\right) .
$$

The choices for the gluings of $L_{1}$ and $L_{2}$ over $n_{6}$ and $n_{7}$ yield $r$ distinct limit roots $\xi_{i}=\left(X, L_{i}\right)$ of $\left(C, \omega_{C}\right), i=\{1, \ldots, r\}$. Now consider a general one-parameter smoothing $\left(f_{i}: \mathcal{X} \rightarrow S, \mathcal{L}_{i}\right)$ of $\left(X, L_{i}\right)$. The surface $\mathcal{X}$ is smooth at $q_{1}, \ldots, q_{5}, n_{6}, n_{7}$ and has $A_{r-2}$ singularities at $p_{1}, \ldots, p_{5}$; therefore $C_{2}$ is a Cartier divisor. The line bundle $\mathcal{M}_{i}:=\mathcal{L}_{i} \otimes \mathcal{O}_{\mathcal{X}}\left(-C_{2}\right)$ has degree zero on all exceptional components of $X$, hence it descends to $\mathcal{M}_{i}^{\prime} \in \operatorname{Pic} \mathcal{C}$, where $\mathcal{C} \rightarrow S$ is the stable model of $\mathcal{X} \rightarrow S$. Now set $M_{i}:=\left(\mathcal{M}_{i}^{\prime}\right)_{\mid C}$. Then $M_{i}$ is stably balanced and determines a point $\lambda_{i} \in \widehat{S}_{C}^{r, 1}$. Observe that $M_{i}^{\otimes r} \simeq \omega_{C} \otimes T_{i}$, where $T_{i}$ is a twister on $C$ and

$$
\left(T_{i}\right)_{\mid C_{1}} \simeq \mathcal{O}_{C_{1}}\left(-\sum_{j=1}^{5} p_{j}\right), \quad\left(T_{i}\right)_{\mid C_{2}} \simeq \mathcal{O}_{C_{2}}\left(\sum_{j=1}^{5} q_{j}\right) .
$$

Then, as in the proof of Proposition 5.3.3, we see that there is a 6-dimensional irreducible component $Z$ of $\widehat{S}_{C}^{r, 1}$ containing $\lambda_{1}, \ldots, \lambda_{r}$. Moreover, as we vary the families $f_{i}$, the points $\lambda_{i}$ cover an open subset of $Z$. This shows that $Z$ is entirely contained in the subscheme where $\chi^{-1}$ is not defined. Since the curve $C$ itself varies in a codimension 7 stratum of $\bar{M}_{g}$, we obtain that $\chi^{-1}$ is not regular over a codimension 1 subscheme of $\widehat{S}_{g}^{r, 1}$.

Our comparison results extend easily to torsion-free sheaves compactifications. More precisely, recall that Pandharipande constructs in [20] a compactification $\overline{U_{g}(d, 1)}$ of $P_{d, g}$ over $\bar{M}_{g}$, by means of rank 1 torsion-free sheaves, and shows that this compactification is isomorphic to $\bar{P}_{d, g}$ under the correspondence given by Proposition 4.2.2. Let $R_{g}^{1 / r}\left(\omega^{\otimes l}\right)$ be the coarse moduli space of Jarvis's stack 
$\operatorname{Root}_{g}^{1 / r}\left(\omega^{\otimes l}\right)$ of $r$-th root sheaves of $\omega^{\otimes l}$ over $\bar{M}_{g}$ (see section 4.2). Set $d=$ $l(2 g-2) / r$. Then we have a commutative diagram:

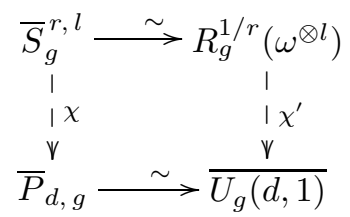

Therefore all results about $\chi$ hold equally for $\chi^{\prime}$. In particular, we have:

Corollary 5.4.3. The coarse moduli spaces of the stacks $\operatorname{RoOT}_{g}^{1 / r}\left(\omega^{\otimes l}\right)$ and $\overline{\mathfrak{S}}_{g}^{1 / r}\left(\omega^{\otimes l}\right)$ (in the notation of [15]) do not embed in $\overline{U_{g}(d, 1)}$.

Proof. For $R_{g}^{1 / r}\left(\omega^{\otimes l}\right)$, the statement is an immediate consequence of Theorem 4.2 .3 and Theorem 5.4.2 (ii). Let $T_{g}^{1 / r}\left(\omega^{\otimes l}\right)$ be the coarse moduli space of the stack $\overline{\mathfrak{S}}_{g}^{1 / r}\left(\omega^{\otimes l}\right)$. Then $T_{g}^{1 / r}\left(\omega^{\otimes l}\right)$ is the normalization of $R_{g}^{1 / r}\left(\omega^{\otimes l}\right)$, so it is finite over $\bar{M}_{g}$. The statement follows.

The literature on compactified Picard varieties is quite rich and varied, so that it would be worthwhile to study the same issue with respect to other compactifications, such as those of [24, [10], [17, for example. This naturally leads to the problem of comparing among each other various constructions of the compactified jacobian; the general situation is not as clear-cut as it is for the two compactifications ([5] and [20]) used in our paper. Recent results in [2] show that, under suitable hypotheses, the spaces of [5] and 24] coincide; other correlation results are, at least to us, not known.

We conclude with a detailed example.

Example 5.4.4. Let $C$ be as in Example 4.1.5. Assume also, for simplicity, that $C_{i} \not \mathbb{P}^{1}$ for $i=1,2$. Under this assumption, two limit third roots are isomorphic if and only if the underlying line bundles are isomorphic off the exceptional components. The quasistable curves having stable model $C$ are $X_{i}$, obtained blowing up the node $n_{i}$ for $i=1,2,3$, and $X_{j, k}$ and $X_{1,2,3}$, described in Example 4.1.5. The balanced multidegrees are:

$$
\begin{aligned}
& \circ \underline{d}=\left(d_{C_{1}}, d_{C_{2}}\right)=(1,-1),(0,0) \text { and }(-1,1) \text { on } C ; \\
& \circ \underline{d}=\left(d_{C_{1}}, d_{C_{2}}, d_{E_{i}}\right)=(0,-1,1) \text { and }(-1,0,1) \text { on } X_{i} ; \\
& \circ \underline{d}=\left(d_{C_{1}}, d_{C_{2}}, d_{E_{j}}, d_{E_{k}}\right)=(-1,-1,1,1) \text { on } X_{j, k} .
\end{aligned}
$$

All these degrees are stably balanced; no multidegree on $X_{1,2,3}$ is balanced.

Let $\bar{P}_{C}^{0}$ be the compactified jacobian of $C$. Recall from [5], $\S 7.3$, that $\bar{P}_{C}^{0}$ is the union of strata $V_{I}^{d}$ parametrized by the balanced multidegrees listed above. Each stratum $V_{I}^{\underline{d}}$ is isomorphic to the variety of line bundles of multidegree $\underline{d}$ on the quasistable curve obtained blowing-up the nodes $\left\{n_{i}\right\}_{i \in I}$ of $C$ (we denote by $V^{\underline{d}}$ the strata $\left.V_{\bar{\emptyset}} \frac{d}{}\right)$. The three open strata $V^{(1,-1)}, V^{(0,0)}$ and $V^{(-1,1)}$ are $\left(\mathbb{C}^{*}\right)^{2}$-bundles over $\mathrm{Pic}^{1} C_{1} \times \mathrm{Pic}^{-1} C_{2}, \mathrm{Pic}^{0} C_{1} \times \mathrm{Pic}^{0} C_{2}$ and $\mathrm{Pic}^{-1} C_{1} \times \mathrm{Pic}^{1} C_{2}$ respectively; their closures are the irreducible components of $\bar{P}_{C}^{0}$. In $\bar{V}^{(1,-1)}$ and $\bar{V}^{(-1,1)}$, the fibers are compactified by $\mathbb{P}^{2}$; in $\bar{V}^{(0,0)}$ they are compactified by $\mathbb{P}^{2}$ blown-up in three points. 
These three irreducible components intersect along the codimension 1 strata; see [5], page 651, for more details.

Recall that $g^{\nu}=g-2$; we are going to show that $\widehat{S}_{C}^{3,0}$ consists of $15 \cdot 3^{2 g^{\nu}}$ points and $2 \cdot 3^{2 g^{\nu}}$ disjoint copies of $\mathbb{P}^{2}$.

Among the points, $9 \cdot 3^{2 g^{\nu}}$ lie in $V^{(0,0)}$, and are the $L \in \operatorname{Pic} C$ with $L^{3} \simeq \mathcal{O}_{C}$; the remaining $6 \cdot 3^{2 g^{\nu}}$ are the $L \in \operatorname{Pic} X_{j, k}$ with $L_{\mid \widetilde{X}_{j, k}}^{\otimes 3} \simeq \mathcal{O}_{\widetilde{X}_{j, k}}\left(-2 p_{j}-q_{j}-p_{k}-2 q_{k}\right)$ or $L_{\mid \widetilde{X}_{j, k}}^{\otimes 3} \simeq \mathcal{O}_{\widetilde{X}_{j, k}}\left(-p_{j}-2 q_{j}-2 p_{k}-q_{k}\right)$; they lie in $V_{j, k}^{(-1,-1,1,1)}$. All these points are limit roots and lie also in $\bar{S}_{C}^{3,0}$; note that the remaining points in $\bar{S}_{C}^{3,0}$ have unbalanced multidegree.

Each $\mathbb{P}^{2}$ in $\widehat{S}_{C}^{3,0}$ corresponds to a choice of $L_{1} \in \operatorname{Pic} C_{1}$ and $L_{2} \in \operatorname{Pic} C_{2}$ such that either $L_{1}^{\otimes 3} \simeq \mathcal{O}_{C_{1}}\left(p_{1}+p_{2}+p_{3}\right)$ and $L_{2}^{\otimes 3} \simeq \mathcal{O}_{C_{2}}\left(-q_{1}-q_{2}-q_{3}\right)$, or conversely. We describe the first case, the other being symmetric. So, fix such $L_{1}$ and $L_{2}$. Note that $\left(L_{1}, L_{2}\right) \in \mathrm{Pic}^{1} C_{1} \times \mathrm{Pic}^{-1} C_{2}$. Then the corresponding $\mathbb{P}^{2}$ is the fiber over $\left(L_{1}, L_{2}\right)$ of the $\mathbb{P}^{2}$-bundle $h: \bar{V}^{(1,-1)} \rightarrow \mathrm{Pic}^{1} C_{1} \times \mathrm{Pic}^{-1} C_{2}$. This $\mathbb{P}^{2}$ contains three types of points:

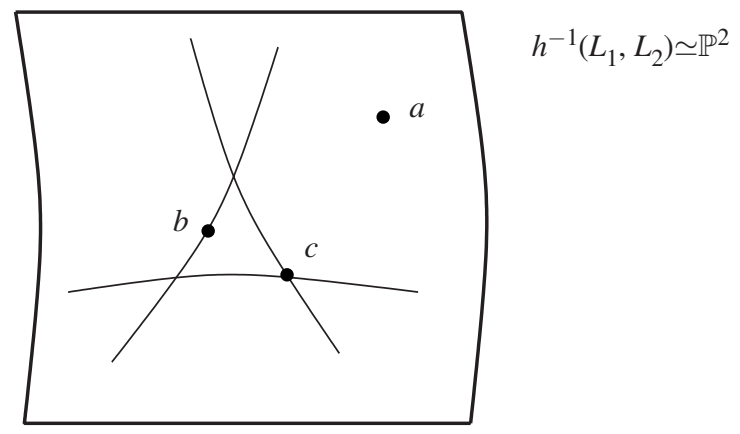

The points in $V^{(1,-1)}$ ( $\alpha$ in the figure) correspond to the $L \in \operatorname{Pic} C$ with $L_{\mid C_{1}} \simeq L_{1}$ and $L_{\mid C_{2}} \simeq L_{2}$; the open stratum $h^{-1}\left(L_{1}, L_{2}\right) \cap V^{(1,-1)}=\left(\mathbb{C}^{*}\right)^{2} \subset \mathbb{P}^{2}$ parametrizes the gluing data for $L_{1}$ and $L_{2}$ over $C$. These points are obtained as the central fiber of a 1-parameter family $(\mathcal{C} \rightarrow S, \mathcal{L})$, where $\mathcal{C}$ is smooth and $\mathcal{L} \in \operatorname{Pic} \mathcal{C}$ is such that $\mathcal{L}^{\otimes 3} \simeq \mathcal{O}_{\mathcal{C}}\left(-C_{1}\right)$. The line bundle $\mathcal{O}_{\mathcal{C}}\left(-C_{1}\right)_{\mid C}$ depends on the family.

The points in $V_{1}^{(0,-1,1)} \cup V_{2}^{(0,-1,1)} \cup V_{3}^{(0,-1,1)}(\beta$ in the figure) are the $L \in$ Pic $X_{i}$ with $L_{\mid C_{1}} \simeq L_{1} \otimes \mathcal{O}_{C_{1}}\left(-p_{i}\right)$ and $L_{\mid C_{2}} \simeq L_{2}$. For each $i$, the open stratum $h^{-1}\left(L_{1}, L_{2}\right) \cap V_{i}^{(0,-1,1)} \simeq \mathbb{C}^{*} \subset \mathbb{P}^{2}$ parametrizes the gluing data for $L_{1} \otimes \mathcal{O}_{C_{1}}\left(-p_{i}\right)$ and $L_{2}$ over $\widetilde{X}_{i}$. These points are obtained as the central fiber of a 1-parameter family $\left(\mathcal{X}_{i} \rightarrow S, \mathcal{L}\right)$, where $\mathcal{X}_{i}$ has an $A_{2}$ singularity in $q_{i}$ and $\mathcal{L} \in \operatorname{Pic} \mathcal{X}_{i}$ is such that $\mathcal{L}^{\otimes 3} \simeq \mathcal{O}_{\mathcal{X}_{i}}\left(-C_{1}-3 E_{i}\right)$.

Finally, the three points in $V_{1,2}^{(-1,-1,1,1)}, V_{1,3}^{(-1,-1,1,1)}$ and $V_{2,3}^{(-1,-1,1,1)}(\gamma$ in the figure) are the $L \in \operatorname{Pic} X_{j, k}$ with $L_{\mid C_{1}} \simeq L_{1} \otimes \mathcal{O}_{C_{1}}\left(-p_{j}-p_{k}\right)$ and $L_{\mid C_{2}} \simeq L_{2}$. They are obtained as the central fiber of a 1-parameter family $\left(\mathcal{X}_{j, k} \rightarrow S, \mathcal{L}\right)$, 
where $\mathcal{X}_{j, k}$ has $A_{2}$ singularities in $q_{j}$ and $q_{k}$, and $\mathcal{L} \in \operatorname{Pic} \mathcal{X}_{j, k}$ is such that $\mathcal{L}^{\otimes 3} \simeq$ $\mathcal{O}_{\mathcal{X}_{j, k}}\left(-C_{1}-3 E_{j}-3 E_{k}\right)$.

\section{REFERENCES}

[1] Dan Abramovich and Tyler J. Jarvis. Moduli of twisted spin curves. Proceedings of the American Mathematical Society, 131(3):685-699, 2002. MR1937405(2003k:14027)

[2] Valery Alexeev. Compactified Jacobians and Torelli map. Publ. RIMS, Kyoto University 40:1241-1265, 2004. MR2105707 (2006a:14016)

[3] Edoardo Ballico, Cinzia Casagrande, and Claudio Fontanari. Moduli of Prym curves. Documenta Mathematica, 9:265-281, 2004. MR2117416 (2006e:14031)

[4] Arnaud Beauville. Prym varieties and the Schottky problem. Inventiones Mathematicae, 41:149-196, 1977. MR0572974 (58:27995)

[5] Lucia Caporaso. A compactification of the universal Picard variety over the moduli space of stable curves. Journal of the American Mathematical Society, 7(3):589-660, 1994. MR.1254134 (95d:14014)

[6] Lucia Caporaso and Cinzia Casagrande. Combinatorial properties of stable spin curves. Communications in Algebra, 31(8):3653-3672, 2003. Special Issue in Honor of Steven L. Kleiman. MR2007379 (2004i:14026)

[7] Alessandro Chiodo. Higher Spin curves and Witten's top Chern class. Ph.D. thesis, University of Cambridge, 2003.

[8] Maurizio Cornalba. Moduli of curves and theta-characteristics. In Lectures on Riemann Surfaces: Proceedings of the College on Riemann Surfaces, International Centre for Theoretical Physics, Trieste, 1987, pages 560-589. World Scientific, 1989. MR1082361 (91m:14037)

[9] Maurizio Cornalba. A remark on the Picard group of spin moduli space. Rendiconti Lincei. Matematica e Applicazioni, 2:211-217, 1991. MR1135424 (92k:14007)

[10] Eduardo Esteves. Compactifying the relative Jacobian over families of reduced curves. Transactions of the American Mathematical Society, 353(8):3045-3095, 2001. MR 1828599 (2003b:14036)

[11] Gerd Faltings. Moduli-stacks for bundles on semistable curves. Mathematische Annalen, 304:489-515, 1996. MR1375622 (97d:14016)

[12] Claudio Fontanari. On the geometry of the compactification of the universal Picard variety. To appear in Rendiconti Lincei. Matematica e Applicazioni, 2005.

[13] David Gieseker. Lectures on moduli of curves. Tata Institute of Fundamental Research Lectures on Mathematics and Physics, 69. Springer-Verlag, Berlin, New York, 1982. MR0691308 (84h:14035)

[14] Tyler J. Jarvis. Torsion-free sheaves and moduli of generalized spin curves. Compositio Mathematica, 110:291-333, 1998. MR.1602060 (99b:14026)

[15] Tyler J. Jarvis. Geometry of the moduli of higher spin curves. International Journal of Mathematics, 11(5):637-663, 2000. MR:1780734 (2001f:14050)

[16] Tyler J. Jarvis. The Picard group of the moduli of higher spin curves. New York Journal of Mathematics, 7:23-47, 2001. MR.1838471 (2002j:14032)

[17] Tyler J. Jarvis. Compactification of the universal Picard over the moduli of stable curves. Math. Z., 235(1):123-149, 2001. MR 1785075 (2001m:14040)

[18] Yuan-Pin Lee. Witten's conjecture and Virasoro conjecture for genus up to two. Preprint math.AG/0310442.

[19] David Mumford, John Fogarty, and Frances Clare Kirwan. Geometric Invariant Theory, volume 34 of Ergebnisse der Mathematik und ihrer Grenzgebiete. Springer-Verlag, third edition, 1994. First edition 1965. MR.1304906 (95m:14012)

[20] Rahul Pandharipande. A compactification over $\overline{M_{g}}$ of the universal moduli space of slopesemistable vector bundles. Journal of the American Mathematical Society, 9(2):425-471, 1996. MR:1308406 (96f:14014)

[21] Alexander Polishchuk. Moduli spaces of curves with effective r-spin structures. Preprint math.AG/0309217

[22] Alexander Schmitt. The Hilbert compactification of the universal moduli space of semistable vector bundles over smooth curves. Journal of Differential Geometry, 66:169-209, 2004. MR2106123 (2005j:14044) 
[23] Conjeeveram S. Seshadri. Degenerations of the moduli spaces of vector bundles on curves. In Moduli Spaces in Algebraic Geometry - School on Algebraic Geometry (Trieste, 1999), volume 1 of ICTP Lecture Notes Series, pages 205-265, 2000. MR1795864 (2001i:14044)

[24] Carlos T. Simpson. Moduli of representations of the fundamental group of a smooth projective variety. Inst. Hautes Études Sci. Publ. Math., 80:5-79, 1994. MR.1320603 (96e:14013)

Dipartimento di Matematica, Università Roma Tre, Largo S. L. Murialdo, 1, 00146 Roma, ITALY

E-mail address: caporaso@mat.uniroma3.it

Dipartimento di Matematica "L. Tonelli", Università di Pisa, Largo B. Pontecorvo, 5, 56127 Pisa, ItAly

E-mail address: casagrande@dm.unipi.it

Dipartimento di Matematica "F. Casorati", Università di Pavia, Via Ferrata, 1, 27100 PAVIA, ITALY

E-mail address: maurizio.cornalba@unipv.it 